Revue internationale P.M.E.

\title{
La responsabilité sociale dans les achats : facteurs explicatifs, diffusion et changements apportés. Le cas de trois enseignes du secteur de l'ameublement \\ Socially responsible buying policies : main factors, diffusion and changes brought. The case of three companies in the furniture industry \\ Políticas de RSE en las compras : principales factores explicativos, diffusión, y cambios traídos. El caso de tres enseñas del sector mobiliario
}

Dominique Casin

Volume 30, numéro 3-4, 2017

URI : https://id.erudit.org/iderudit/1042665ar

DOI : https://doi.org/10.7202/1042665ar

Aller au sommaire du numéro

Éditeur(s)

Editions EMS - In Quarto SARL

ISSN

0776-5436 (imprimé)

1918-9699 (numérique)

Découvrir la revue

Citer cet article

Casin, D. (2017). La responsabilité sociale dans les achats : facteurs explicatifs, diffusion et changements apportés. Le cas de trois enseignes du secteur de l'ameublement. Revue internationale P.M.E., 30(3-4), 191-230.

https://doi.org/10.7202/1042665ar

\section{Résumé de l'article}

La globalisation de l'économie a conduit les grandes entreprises à constituer autour d'elles des réseaux de fournisseurs et de sous-traitants. Cette démarche leur permet de répondre à la complexité de leur environnement, mais vise également un objectif stratégique de recherche de partenaires. Parallèlement, depuis les années 1990, on assiste au développement de la notion de responsabilité sociale des entreprises (RSE) en réaction aux pressions exercées par les différentes parties prenantes. Le management des entreprises est devenu par conséquent plus complexe puisqu'il doit concilier à la fois des exigences économiques et des exigences sociales. À partir d'une étude qualitative réalisée auprès de trois enseignes du secteur de l'ameublement et d'une enquête par questionnaire concernant leurs sous-traitants (en grande majorité des PME d'origine asiatique et/ou européenne), cet article se propose d'analyser les facteurs explicatifs des politiques d'achats socialement responsables ainsi que les changements apportés par ces démarches chez ces donneurs d'ordre et chez leurs sous-traitants. Cette recherche fait ressortir le rôle clé joué par le type de relation de sous-traitance dans les stratégies RSE des donneurs d'ordre. Elle apporte également un éclairage sur les pratiques qui améliorent la performance des fournisseurs. 


\title{
La responsabilité sociale dans les achats: facteurs explicatifs, diffusion et changements apportés. Le cas de trois enseignes du secteur de l'ameublement
}

\begin{abstract}
Dominique CASIN
Dominique Casin est docteure en sciences de gestion et enseigne l'économie et le marketing à l'Université de Lorraine (Langues étrangères appliquées, Metz). Ses recherches portent sur les politiques RSE des grands groupes et leurs relations avec leurs fournisseurs et sous-traitants.

\author{
Université de Lorraine \\ UFR ALL, Département LEA \\ Île du Saulcy \\ 57045 METZ CEDEX 01, France
} dominique.casin@univ-lorraine.fr
\end{abstract}

\begin{abstract}
RÉSUMÉ
La globalisation de l'économie a conduit les grandes entreprises à constituer autour d'elles des réseaux de fournisseurs et de sous-traitants. Cette démarche leur permet de répondre à la complexité de leur environnement, mais vise également un objectif stratégique de recherche de partenaires. Parallèlement, depuis les années 1990, on assiste au développement de la notion de responsabilité sociale des entreprises (RSE) en réaction aux pressions exercées par les différentes parties prenantes. Le management des entreprises est devenu par conséquent plus complexe puisqu'il doit concilier à la fois des exigences économiques et des exigences sociales. À partir d’une étude qualitative réalisée auprès de trois enseignes $d u$ secteur de l'ameublement et d'une enquête par questionnaire concernant leurs sous-traitants (en grande majorité des PME d'origine asiatique et/ou européenne), cet article se propose d'analyser les facteurs explicatifs des politiques d'achats socialement responsables ainsi que les changements apportés par ces démarches chez ces donneurs d'ordre et chez leurs sous-traitants. Cette recherche fait ressortir le rôle clé joué par le type de relation de sous-traitance dans les stratégies RSE des donneurs d'ordre. Elle apporte également un éclairage sur les pratiques qui améliorent la performance des fournisseurs.
\end{abstract}

Mots-CLÉs

Politiques RSE dans les achats, Facteurs. Changements, Relations de sous-traitance, PME 


\title{
Socially responsible buying policies: main factors, diffusion and changes brought. The case of three companies in the furniture industry
}

\begin{abstract}
Over the last twenty years, the globalization of the economy has led companies to develop extensive networks of suppliers and subcontractors. Since the 1990's, the globalization and the liberalization of economic activity have been gone hand in hand with the development of Corporate Social Responsibility (CSR) due to pressure from the different stakeholders. In the face of such evolutions, the firms'management has become more complex because of the combination of economic and social objectives. We study the CSR policies conducted by three companies in the furniture industry. From a qualitative study and a survey, this paper proposes to highlight the main factors of Socially Responsible Buying (SRB) Policies and the changes caused by these policies in these companies and their SME subcontactors. This research shows that type of subcontracting relation is a key factor to explain CSR strategies of the buyers. It also highlights what practices improve supplier performance.
\end{abstract}

KEYWORDS

Socially responsible buying (SRB) Policies, Factors, Changes, Subcontracting relations. SMEs

\section{Politicas de RSE en las compras : principales factores explicativos, diffusión, y cambios traidos. El caso de tres enseñas del sector mobiliario}

\begin{abstract}
RESUMEN
La globalización en la economía ha llevado las grandes empresas, esos últimos veinte años, a recurrir a redes extendidas de proveedores y subcontratistas. En paralelo, desde los años 90 asistimos al desarrollo de la idea de RSE (responsabilidad social de las empresas) en reacción a las presiones ejercidas por las diferentes partes interesadas. Frente a estas evoluciones, la gestión de empresas se ha vuelto más compleja ya que debe compaginar a la vez exigencias económicas y exigencias sociales. Estudiaremos las políticas de RSE en las compras llevadas a cabo por tres enseñas del sector mobiliario. Este artículo, a partir de un estudio cualitativo y de una encuesta, propone destacar los principales factores explicativos de las políticas de compras socialmente responsables y los cambios traídos por estas políticas a nivel de esas enseñas $y$ de sus prooedores PyME.
\end{abstract}

Palabras clave

Politicas de RSE en las compras, Factores, Cambios, Relaciones de subcontratación, PyME 


\section{INTRODUCTION}

La mondialisation a bouleversé de manière importante l'organisation de la production, les marchés financiers et les régulations propres au système fordiste (Gendron, Lapointe et Turcotte, 2004). Les grandes entreprises se sont recentrées sur leur métier principal et ont restructuré les relations avec leurs fournisseurs et sous-traitants. L'entreprise intégrée et hiérarchisée a cédé la place à l'entreprise réseau (Baudry, 1995 ; Mariotti, Reverdy et Segrestin, 2001 ; Billaudot et Julien, 2003). Parallèlement à ces transformations, et face aux pressions de leurs différentes parties prenantes (actionnaires, salariés, clients, ONG, fournisseurs, pouvoirs publics), les grands groupes ont dû se positionner face aux questionnements relatifs à la RSE. Les achats responsables sont devenus un «standard » pour ces derniers (ORSE/Ecovadis, 2010).

Bien que le développement durable fasse l'objet d'une abondante littérature, la dimension sociale dans les achats est une problématique relativement nouvelle et encore peu explorée (Quairel et Auberger, 2007 ; Belin-Munier, 2009 ; Leire et Mont, 2010 ; Fel, 2011 ; Brandeburg, Govindan, Sarkis et Seuring, 2014). La recherche est largement dominée par les préoccupations environnementales (Seuring et Müller, 2008 ; Hajmohammad, Vachon, Klassen et Gavronski, 2013).

Comme le mettent en avant un certain nombre d'auteurs (Dupuis, 2008 ; Belin-Munier, 2009 ; Vercher, 2010 ; Acquier, Daudigeos et Valiorgue, 2011 ; Baudry et Chassagnon, 2012 ; Frigant, 2012), le développement des " firmes-réseaux » transnationales nécessite d'étudier la question de la responsabilité sociale sous l'angle non pas d'une seule entreprise, mais " d'un ensemble d'entreprises imbriquées dans une chaîne de valeur éclatée ${ }^{1}$ " pour reprendre les termes d'Acquier, Daudigeos et Valiorgue, 2011. C'est donc sous cet angle, et en prenant appui sur les politiques menées par trois enseignes du secteur de l'ameublement que nous aborderons la problématique de la RSE dans les achats. Dans ce secteur, selon une étude du ministère de l'Économie, des Finances et de l'Industrie - SESSI - (Marbach, 2007), «le distributeur n'est plus un simple acheteur-revendeur. Il devient un véritable donneur d'ordres qui externalise la production chez l'industriel ». Le client précise les spécificités fonctionnelles du produit, mais c'est le vendeur appelé sous-traitant/fournisseur (Baudry, 1995) qui assure la conception du produit. De ce fait, le qualificatif qui convient ici est celui de sous-traitants.

En prenant appui sur une étude qualitative réalisée auprès de ces trois donneurs d'ordre (DO), sur une enquête par sondage menée auprès de leurs sous-traitants PME, nous nous proposons de répondre aux questions suivantes : quels sont les facteurs explicatifs des politiques d'achats socialement responsables? Quels sont les changements apportés par ces démarches RSE chez les DO et chez leurs sous-traitants? Dans une première partie, nous exposerons le cadre théorique relatif à la RSE en général et dans les achats ainsi que le modèle théorique de départ (Carter, 2005). Puis, nous présenterons l'étude qualitative et ses résultats. Ensuite, nous mobiliserons ces apports pour compléter le modèle initial et proposerons un modèle explicatif des politiques d'achats socialement responsables. Ce dernier servira de base au questionnaire fournisseurs (Annexe 1). Enfin nous présenterons l'enquête par sondage et ses principaux résultats.

1 Une chaîne de valeur est « un ensemble d'entreprises juridiquement indépendantes reliées entre elles par des opérations marchandes plus ou moins récurrentes d'approvisionnement, de production et de distribution» (Acquier, Daudigeos et Valiorgue, 2011, p. 168). 


\section{LE CADRE THÉORIQUE}

\subsection{Le concept de responsabilité sociale des entreprises}

C'est à Bowen que l'on doit, en 1953, les premières formalisations du concept de RSE. Les décisions des hommes d'affaires doivent aller dans le sens des orientations et des valeurs souhaitées par la société. C’est une conception fondée sur l'éthique.

Par la suite, le développement des travaux sur la RSE voit s'opposer les défenseurs d'une responsabilité élargie de l'entreprise à l'égard de la société et ceux pour lesquels (Levitt, 1958 ; Friedman, 1970) l'entreprise n'a d'autre responsabilité que la recherche d'un profit maximum.

Le milieu des années 60 constitue un tournant pour l'image de l'entreprise dans la société et la décennie suivante est marquée par des mouvements sociaux et environnementaux prenant souvent pour cible les entreprises. Apparaît alors une approche managériale de la RSE visant à étudier les modes de réaction des entreprises aux pressions externes issues de leur environnement (Ackermann et Bauer, 1976).

À la suite de ces travaux, au cours des années 80 et 90, la conception utilitariste de la RSE s'impose largement. Les recherches se portent sur les résultats des politiques RSE (concept de performance sociale de l'entreprise) et sur les groupes envers lesquels l'entreprise exerce, ou devrait exercer, ses responsabilités sociales. La RSE s'enrichit alors du concept de parties prenantes (Freeman, 1984). Dans le paradigme utilitaire-stratégique [selon la typologie de Gendron (2000)], le comportement social de l'entreprise doit lui permettre d'améliorer sa performance financière. Les discours insistent alors sur le caractère "gagnant-gagnant » des stratégies RSE pour les entreprises et leurs parties prenantes ("business case »).

Dans les années 2000, la mondialisation des marchés et les stratégies d'externalisation des entreprises conduisent au développement de firmes-réseaux (Baudry et Chassagnon, 2012). On voit également émerger et monter en puissance de nouveaux acteurs (associations de consommateurs, ONG par exemple), très vigilants sur les stratégies des grandes entreprises et qui vont participer au développement des démarches RSE. La question de la responsabilité sociale de l'entreprise doit donc s'examiner dans ce nouveau contexte et concerne l'ensemble de la chaîne d'approvisionnement.

En réponse à ces changements, de nombreuses initiatives voient le jour au niveau européen et international. Il s'agit notamment du Livre vert de la Commission de l'Union européenne (2001) qui met l'accent sur les démarches volontaires des entreprises dans le domaine social, environnemental, des droits de l'homme. Le Global Compact ou Pacte mondial des Nations Unies (1999) appelle les entreprises à promouvoir et à respecter dix principes relatifs aux droits de l'homme, aux conditions de travail, à l'environnement et à la lutte contre la corruption. L'intégration de la chaîne de fournisseurs dans la sphère de responsabilité sociale des entreprises fait partie des principes directeurs de l'OCDE, révisés en 2011. On note enfin un travail important de normalisation dans le domaine de la RSE avec en particulier pour la fonction achats, la norme internationale ISO 26000 (publiée en novembre 2010) et en France celle de l'AFNOR (FD X50-135, publiée en juillet 2012). 


\subsection{La responsabilité sociale dans les achats}

Après avoir défini le concept de responsabilité sociale dans les achats, nous exposerons les principaux facteurs explicatifs, puis nous présenterons le modèle théorique de départ (Carter, 2005).

\subsubsection{LA DÉFINITION DU CONCEPT DE RESPONSABILITÉ SOCIALE DANS LES ACHATS}

Plusieurs auteurs ont défini le concept de responsabilité sociale dans les achats ou achats responsables, politiques d'achats responsables, management durable de la chaîne d'approvisionnement (Maignan et Ralston, 2002 ; Carter et Jennings, 2002, 2004 ; Seuring et Müller, 2008 ; Seuring, Sarkis, Müller et Rao, 2008 ; Salam, 2009 ; Leire et Mont, 2010).

Pour Carter et Jennings (2004), les dimensions suivantes sont concernées : l'environnement, l'éthique (contrats clairs, pas de favoritisme dans la sélection des fournisseurs), la diversité (achats auprès de minorités), les droits de l'homme et la sécurité dans les usines des fournisseurs, la philanthropie (contributions et engagement dans la communauté).

Selon Seuring et Müller (2008), le management durable de la chaîne d'approvisionnement concerne la gestion des flux physiques, d’information, financiers, mais aussi la coopération entre entreprises intégrant des objectifs économiques, environnementaux et sociaux issus des attentes des parties prenantes.

Un certain nombre de travaux traitent de la dimension sociale, dimension privilégiée dans cet article. Carter et Jennings (2002) évoquent les actions, dans le domaine des droits de l'homme, attendues d'une entreprise s'engageant en matière de RSE : veiller à ce que ses fournisseurs ne fassent pas travailler des enfants, à ce que leurs usines ne soient pas des lieux d'exploitation de la main-d'œuvre ("sweatshops») ou encore les encourager à verser à leurs salariés des rémunérations supérieures au salaire minimum local. Les achats socialement responsables désignent un ensemble de problématiques liées aux droits de l'homme principalement, à la sécurité, à la diversité, à la philanthropie et à la communauté (Carter, 2004 ; Leire et Mont, 2010) et incluent les droits des travailleurs, les salaires, l'insertion des travailleurs handicapés, l'égalité entre les sexes, raciale et ethnique (Lobel, 2006 ; Leire et Mont, 2010). Carter (2005) évoque aussi le développement des fournisseurs (visites d'usines, audits et formation). L'entreprise cliente peut aider ces derniers à améliorer leurs processus de production, leurs emballages, leurs produits en termes d'initiatives environnementales. Elle peut également travailler avec des petits fournisseurs minoritaires pour accroître leur compétitivité et leur qualité. Enfin, les acheteurs ont la possibilité de visiter puis certifier les fournisseurs respectant les critères liés aux droits de l'homme.

Les initiatives des grands organismes internationaux permettent également de mieux cerner les pratiques sociales dans les achats. En 2010, l'ONU a édité un guide pour une démarche proactive au niveau de la chaîne d’approvisionnement (révisé en 2015). Sur son site Internet sont décrites les pratiques responsables d'entreprises signataires. On y retrouve les thèmes évoqués précédemment : audits suivis de plans d'actions correctrices, diffusion des pratiques RSE par les fournisseurs ou encore la formation. La Fondation européenne pour l'amélioration des conditions de vie et de travail (Segal, Sobczak et Triomphe, 2003) dresse un 
inventaire de ces pratiques : coordination, suivi par le DO des initiatives en matière de sécurité au travail ; contribution à la formation du personnel ; aide concrète apportée aux soustraitants dans la démarche de normalisation et de labellisation de leurs pratiques en matière de qualité, d'environnement ; reconnaissance de cette responsabilité sociale envers toute la filière de production ; pratiques d'achat favorisant la mise en œuvre de progrès sociaux chez les fournisseurs ; prise en compte des critères sociaux pour l'évaluation des prestations et des contrats.

À ces initiatives publiques s'ajoutent des initiatives privées permettant d'appréhender la performance sociale du fournisseur comme la notation fournisseur développée par Ecovadis ou la grille commune de l'ICS (Initiative Clause Sociale) qui sert de référence aux distributeurs pour leurs codes de conduite et chartes.

\subsubsection{LES FACTEURS EXPLICATIFS DES POLITIQUES D'ACHATS SOCIALEMENT RESPONSABLES}

S’appuyant sur la synthèse de 191 articles, Seuring et Müller (2008) sont les seuls auteurs à faire apparaître par ordre d'importance les facteurs explicatifs des politiques d'achats responsables : la réglementation, les demandes des clients, la réponse aux parties prenantes (respectivement 99, 96 et 90 articles), la recherche d'un avantage concurrentiel (71 articles), les groupes de pression sur le plan environnemental et social et enfin le risque de réputation (respectivement 38 et 30). Un certain nombre de chercheurs mettent également en évidence d'autres facteurs comme la culture organisationnelle, les valeurs ; le leadership des dirigeants ; le type de relation acheteur-fournisseur.

Les pressions des parties prenantes sont largement évoquées dans la littérature (Maignan, Hillebrand et McAlister, 2002 ; Maignan et Ralston, 2002 ; Roberts, 2003 ; Carter et Jennings, 2004 ; Quairel, 2006, 2007 ; Seuring et Müller, 2008 ; Worthington, Ram, Boyal et Shah, 2008 ; Salam, 2009). Ces pressions sont primordiales dans les démarches proactives (Maignan, Hillebrand et McAlister, 2002). Les parties prenantes sont diverses : gouvernements, organisations professionnelles, communauté, médias, parties prenantes organisationnelles (consommateurs, employés, actionnaires, fournisseurs). Les consommateurs jouent un rôle important (Carter et Jennings, 2004 ; Salam, 2009), ces derniers étant de plus en plus sensibles à la sécurité, l'impact environnemental et l'origine des produits. Les entreprises peuvent également chercher à répondre aux attentes des parties prenantes. L'étude comparative réalisée par Maignan et Ralston (2002) montre notamment que pour les entreprises françaises les initiatives RSE sont principalement motivées par ce facteur.

La culture organisationnelle est définie par Carter et Jennings (2004) comme un ensemble de valeurs, de croyances, de suppositions et de manières de penser, partagé par les membres d'une organisation et enseigné aux nouveaux membres. Si elle est " orientée vers les autres ", elle conduira à rechercher le bien-être de ces derniers, à les soutenir et à être équitable. Elle a un effet positif sur le degré d'engagement dans les activités et les programmes socialement responsables et notamment dans les achats (Carter et Jennings, 2004 ; Salam, 2009). Maignan, Hillebrand et McAlister (2002) quant à eux insistent plutôt sur les valeurs organisationnelles qui constituent le niveau fondamental de la culture organisationnelle, ses fondements. Même sans pressions des parties prenantes, les entreprises peuvent malgré tout adopter une démarche proactive, car leurs valeurs organisationnelles dictent de telles pratiques. 
Le leadership des dirigeants représente également un facteur clé (Maignan, Hillebrand et McAlister, 2002 ; Roberts, 2003 ; Carter et Jennings, 2004 ; Quairel, 2006, 2007). C'est l'art de conduire une organisation comme une réalité humaine, de communiquer, de motiver son personnel et de convaincre les personnes à accepter le changement (De Woot, 2005). Les comportements des dirigeants et leurs propres actions ont une influence sur la culture organisationnelle de leur entreprise (Carter et Jennings, 2004 ; Maignan, Hillebrand et McAlister, 2002).

Seuring et Müller (2008), Salam (2009) font ressortir le rôle clé joué par les lois et les règlements. Worthington et al. (2008) évoquent des résultats divergents : les évolutions politiques et législatives représentent un facteur majeur dans les initiatives de nombreuses sociétés américaines visant à promouvoir la diversité des fournisseurs alors que cette influence est relativement insignifiante en Grande-Bretagne. L'étude de Carter et Jennings (2004) ne permet pas d'établir de lien significatif entre la réglementation et les politiques d'achats responsables.

La recherche d'avantages est également très citée dans la littérature. Ce type de pratiques s'inscrit dans le courant utilitariste de la RSE. Cette approche est souvent dominante dans les discours des entreprises, des consultants et des organismes chargés de promouvoir la RSE (Capron et Quairel-Lanoizelée, 2007 ; Gond et Igalens, 2008 ; Capron et Petit, 2011). Les démarches socialement responsables permettent : d'éviter les risques d'image et de réputation, de perte de marché (Maignan, Hillebrand et McAlister, 2002 ; Roberts, 2003 ; Seuring et Müller, 2008) ; de stimuler l'innovation (Maignan, Hillebrand et McAlister, 2002 ; Zhu, Sarkis et Lai, 2007) ; d'améliorer la qualité des produits (Vachon et Klassen, 2008) et de se différencier en termes de produits ou de marchés (Maignan, Hillebrand et McAlister, 2002 ; Roberts, 2003) ; de réaliser des économies de coûts (Roberts, 2003). Pour Carter (2005), qui se base sur les travaux de Brown et Dacin (1997) et de Maignan et Ferrel (2004), les actions socialement responsables d'une entreprise contribuent à améliorer son image de marque et sa réputation auprès de ses parties prenantes clés. Du point de vue de l'approche par les ressources, une réputation positive auprès des fournisseurs peut être une source d'avantage concurrentiel. Cette réputation fait partie de ce que Barney (1999) appelle « des capacités socialement complexes ». Porter et Kramer (2006) montrent comment une grande firme peut intégrer la RSE à sa stratégie afin qu'elle soit source d'avantage concurrentiel et développent le concept de création de valeur partagée (Porter et Kramer, 2011). La prise en compte des besoins et attentes des parties prenantes et la coopération active avec ces dernières permettent de conjuguer création de valeur économique et création de valeur sociétale. Les pratiques responsables des multinationales auprès des petits fournisseurs locaux comme le partage de technologie, l'aide au financement, un accès plus facile aux approvisionnements permettent d'améliorer la qualité et la productivité de ces derniers tout en augmentant les revenus. Cette création de valeur partagée peut également provenir de l'implication d'autres acteurs locaux comme les ONG. Le partenariat de Nestlé et de l'ONG Rain Forest, par exemple, a permis de dispenser des formations aux producteurs de café et ainsi d'améliorer la qualité des produits et la productivité des exploitants (Porter et Kramer, 2011). Wothington et al. (2008), font également ressortir l'importance des opportunités économiques dans les initiatives des grandes organisations visant à promouvoir une plus grande diversité chez leurs fournisseurs (petits fournisseurs, minorités ethniques). 
Enfin, un certain nombre d'auteurs mettent l'accent sur le caractère déterminant du type de relation acheteur-fournisseur dans les politiques d'achats responsables (Quairel [2006, 2007], Quairel et Auberger [2007], Leire et Mont [2010]).

Les stratégies RSE adoptées par une même entreprise peuvent être différentes selon les catégories de fournisseurs (Quairel, 2006, 2007 ; Quairel et Auberger, 2007). Si, par exemple, ces derniers sont substituables, non stratégiques, la démarche RSE mise en place par la grande entreprise cliente conduira à des stratégies de conformité purement symboliques. L'instauration de codes de conduite transmis aux fournisseurs (et éventuellement signés par ces derniers, mais sans dispositifs de contrôle) entre dans ce cadre (Capron et QuairelLanoizelée, 2007). Si le client court un risque de réputation (fournisseurs situés dans des pays en développement à législations faibles et où les conditions sociales sont difficiles), alors la démarche RSE conduira à des stratégies de conformité minimale. Il y aura notamment mise en place d'audits chez les fournisseurs et éventuellement rupture des relations avec ceux qui ne respecteraient pas le code de conduite. Les fournisseurs vont alors s'y conformer de manière minimale sans chercher à faire évoluer de façon notable leurs pratiques dans le domaine social (Quairel, 2007). Dans leur étude portant sur les pratiques d'organisations publiques et privées suédoises, Leire et Mont (2010) quant à eux font ressortir des différences significatives entre les organisations. Alors que pour certaines d'entre elles, la majorité des fournisseurs est auditée, pour d'autres seuls les fournisseurs à risque ou un petit nombre de fournisseurs sont concernés.

Afin de mieux faire ressortir les différents facteurs explicatifs des politiques d'achats responsables présents dans la littérature, une catégorisation est proposée dans le tableau 1.

\subsubsection{LE MODÈle THÉORIQUE DE DÉPART : LE MODÈle DE CARTER}

Peu de travaux académiques portent sur la dimension sociale dans les achats (Seuring et Müller, 2008). Carter et Jennings (2004), Carter (2005) comptent parmi les rares chercheurs ayant publié sur ce thème. Carter et Jennings (2004) ont introduit la notion de "purchasing social responsibility» (PSR) ou responsabilité sociale dans les achats et mené une réflexion sur cette thématique d'un point de vue pratique et théorique (Griffis, Autry, Thornton et Brik, 2014). À l'issue d'une enquête menée auprès d'entreprises américaines produisant des biens de consommation, ces auteurs ont mis en lumière les facteurs explicatifs des politiques d'achats responsables : les pressions des consommateurs, la culture organisationnelle, les valeurs des employés (via leurs initiatives) et le leadership des dirigeants (Carter et Jennings, 2004).

Intégrant ces résultats, Carter (2005) développe ensuite un modèle testant la relation entre le niveau de RSE dans les achats et la performance du fournisseur et de l'entreprise cliente. Prenant appui sur la théorie des ressources et plus particulièrement sur les travaux de Barney (1991) et March (1991), Carter (2005) met en avant l'apprentissage organisationnel comme variable médiatrice entre la RSE dans les achats et la performance du fournisseur et de l'entreprise cliente. Celui-ci survient lorsque le savoir est accumulé (dans les procédures, les règles, mais aussi dans les normes moins formelles et les modèles sociaux et de communication) et appris par les membres de l'organisation. Le développement du fournisseur par l'entreprise cliente permet cet apprentissage organisationnel et par conséquent l'amélioration de la performance de celui-ci. Lapprentissage organisationnel fait partie de ces ressources qui peuvent apporter à l'entreprise un avantage concurrentiel. Les dynamiques de 
savoir et d'innovation sont « essentielles à prendre en compte pour les processus de création de ressources et de potentiels de valeur de l'entreprise " (Acquier et Aggeri, 2008). Le test du modèle proposé par Carter (2005) montre que l'apprentissage organisationnel et la performance du fournisseur agissent comme des variables médiatrices entre la RSE dans les achats et les coûts de l'entreprise cliente.

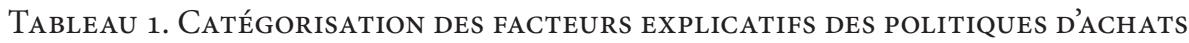 RESPONSABLES}

\begin{tabular}{|c|c|}
\hline Type de Facteurs & Apports \\
\hline $\begin{array}{l}\text { Facteurs culturels } \\
\text { Culture organisationnelle, valeurs } \\
\text { (des employés, des dirigeants) }\end{array}$ & $\begin{array}{l}\text { La RSE est le produit d'une culture. } \\
\text { Influence des facteurs liés à la culture des organisations, à } \\
\text { l'idéologie des dirigeants et à leur conception du rôle de l'en- } \\
\text { treprise dans la société }\end{array}$ \\
\hline $\begin{array}{l}\text { Facteurs économiques } \\
\text { Recherche d'un avantage concur- } \\
\text { rentiel }\end{array}$ & $\begin{array}{l}\text { La RSE dans les achats permet de servir la performance éco- } \\
\text { nomique de l'entreprise (évocation de stratégies « gagnant-ga- } \\
\text { gnant » dans les discours des entreprises et des dirigeants) }\end{array}$ \\
\hline $\begin{array}{l}\text { Facteurs institutionnels } \\
\text { Réglementation } \\
\text { Pressions des parties prenantes }\end{array}$ & $\begin{array}{l}\text { La RSE dans les achats en réponse aux pressions institution- } \\
\text { nelles } \\
\text { Examiner la capacité des acteurs sociaux et des parties pre- } \\
\text { nantes à influencer les entreprises (Gond et Igalens, 2008) } \\
\text { Étude des stratégies des entreprises face à ces contraintes : } \\
\text { conformité, évitement, manipulation (Oliver, 1991) ; stra- } \\
\text { tégies réactives versus proactives (Maignan, Hillebrand et } \\
\text { McAlister, 2002) }\end{array}$ \\
\hline $\begin{array}{l}\text { Facteurs managériaux } \\
\text { Leadership des dirigeants }\end{array}$ & $\begin{array}{l}\text { La RSE dans les achats est influencée par les actions des indi- } \\
\text { vidus et en particulier des dirigeants. Leur rôle est important } \\
\text { dans la mobilisation de tout le personnel et des partenaires de } \\
\text { l'entreprise (Borchani Maktouf, 2010) }\end{array}$ \\
\hline $\begin{array}{l}\text { Réponse aux attentes des parties } \\
\text { prenantes }\end{array}$ & $\begin{array}{l}\text { Pratiques RSE en fonction de l'attention apportée par l'entre- } \\
\text { prise à ses différentes parties prenantes. } \\
\text { Étude des parties prenantes essentielles (hiérarchisation des } \\
\text { parties prenantes : Mitchell, Agle et Wood, 1997) }\end{array}$ \\
\hline $\begin{array}{l}\text { Facteurs organisationnels } \\
\text { Type de relation acheteur- } \\
\text { fournisseur }\end{array}$ & $\begin{array}{l}\text { Différences de pratiques selon la nature des relations avec les } \\
\text { fournisseurs }\end{array}$ \\
\hline
\end{tabular}

Nous nous sommes appuyés sur ce modèle, car celui-ci présente deux contributions significatives à la recherche dans le domaine des politiques d'achats responsables. Ainsi que le souligne Seuring (2013), les différents modèles ne prennent pas en compte le côté social de la RSE et la plupart de ceux testés (Zhu, Sarkis et Lai, 2008 ; Pagell, Krumwiede et Sheu, 2007 ; Vachon et Klassen, 2008 ; Hajmohammad et al., 2013) concernent l'impact des « pratiques vertes » dans la chaîne d'approvisionnement. Carter (2005) fait donc partie des rares auteurs à avoir intégré cet aspect. La deuxième contribution est d'avoir traité la performance comme un concept multidimensionnel. En effet, l'impact des achats responsables sur la per- 
formance du fournisseur est étudié en prenant en compte la qualité, les délais et l'efficacité. L'examen de la performance de la chaîne d'approvisionnement sous ses multiples dimensions est fondamental (Pagell, Krumwiede et Sheu, 2007).

\section{LÉTUDE QUALITATIVE AUPRĖS DE TROIS DONNEURS D'ORDRE DU SECTEUR DE LAMEUBLEMENT}

\subsection{Le choix du domaine d'étude}

Les stratégies RSE menées dans ce secteur sont particulièrement intéressantes à étudier pour trois raisons principales. En premier lieu, le distributeur est devenu un véritable DO qui externalise la production chez l'industriel. Certaines enseignes ont leurs propres designers, les autres interviennent de plus en plus dans la création de modèles chez leurs sous-traitants. Ces DO ont constitué des réseaux de sous-traitants au niveau mondial. En deuxième lieu, c'est un secteur innovant. Les innovations portent sur les produits (comme les produits éco-conçus), les matériaux ou encore les procédés (gestion des flux de marchandises, des flux d'informations). En troisième lieu, c'est un secteur sensible en raison de la matière première utilisée : le bois. Durant les années 80 , les vendeurs de meubles prêts-à-monter sont devenus la cible des campagnes des ONG contre la déforestation, d'où la recherche chez leurs fournisseurs de produits FSC et la mise en ouvre de codes de conduite. Il faut attendre le milieu des années 90 pour que soient prises en considération les conditions de travail chez les fournisseurs (Roberts, 2003).

\subsection{La méthodologie de recherche}

Nous avons retenu la méthode de l'étude de cas, celle-ci étant tout à fait recommandée pour aborder des thématiques nouvelles (Evrard, Pras et Roux 1998) et explorer une situation empirique peu étudiée comme la responsabilité sociale dans les achats. Nous avons choisi de réaliser une étude par cas multiples (Yin, 1984) et de nous intéresser aux démarches RSE de trois enseignes du secteur de l'ameublement. Une telle analyse introduit une dimension de contraste et de comparaison intéressante par rapport au phénomène étudié. La synthèse de la méthodologie de recherche est donnée dans le tableau 2. Nous avons eu recours à l'analyse thématique pour le traitement des données. Celle-ci a été réalisée à partir des thèmes qui ont donné lieu aux rubriques du guide d'entretien. Les données recueillies ont fait l'objet d'une double analyse : verticale et horizontale (un extrait de l'analyse verticale est présenté en annexe 2).

Cette première étape a permis d'explorer les comportements, les motivations qui sous-tendent la démarche RSE des enseignes, de comprendre les stratégies déployées et les changements organisationnels apportés. Elle a aussi contribué à mieux appréhender les relations $\mathrm{DO} /$ soustraitants. Enfin, l'analyse thématique issue de l'étude qualitative a été essentielle pour le développement d'un cadre conceptuel opératoire (prenant appui sur le modèle de Carter, 2005) et la construction du questionnaire administré aux fournisseurs. 
TABleau 2. SynthèSE de LA MÉTHodologie de ReCherche

\begin{tabular}{|c|c|}
\hline Méthodologie & Choix ou positionnement retenus \\
\hline $\begin{array}{l}\text { Stratégie de recherche } \\
\text { pour l'étude qualita- } \\
\text { tive }\end{array}$ & $\begin{array}{l}\text { Méthode de l'étude de cas } \\
\text { - Étude documentaire sur les enseignes de la distribution de meubles } \\
\text { (étude du SESSI, étude du groupe Xerfi², presse spécialisée, sites } \\
\text { Internet des différentes enseignes) } \\
\text { - Recueil de données primaires : entretiens }\end{array}$ \\
\hline Univers d'enquête & $\begin{array}{l}\text { Principales enseignes de la distribution de meubles en France (moins } \\
\text { d'une dizaine sur la base de leur chiffre d'affaires, distribution très } \\
\text { concentrée) }\end{array}$ \\
\hline $\begin{array}{l}\text { Constitution de } \\
\text { l'échantillon }\end{array}$ & $\begin{array}{l}\text { - Critères retenus : chiffre d'affaires, mention d'une démarche RSE } \\
\text { (consultation des sites Internet, revue de presse...) } \\
\text { - Contact de toutes les enseignes. Accord de trois d'entre elles pour } \\
\text { participer à ce travail de recherche (nommées E1, E2 et E3 pour des } \\
\text { raisons de confidentialité) }\end{array}$ \\
\hline $\begin{array}{l}\text { Technique de collecte } \\
\text { de données }\end{array}$ & $\begin{array}{l}\text { - Entretiens semi-directifs (au moins deux dirigeants de chaque en- } \\
\text { seigne, responsables DD et achats, soit au total huit entretiens) } \\
\text { - Déroulement des entretiens par téléphone ou en face à face } \\
\text { - Réalisation des entretiens : mars } 2009 \text { à fin 2010. Jusqu'au début de } \\
\text { l'année 2013, poursuite des contacts avec les dirigeants de chaque } \\
\text { enseigne et aller-retour pour vérifier la convergence des faits et des opi- } \\
\text { nions, clarifier certains points ou recueillir de nouvelles informations }\end{array}$ \\
\hline $\begin{array}{l}\text { Thèmes du guide } \\
\text { d'entretien }\end{array}$ & $\begin{array}{l}\text { Démarche RSE ; relations DO/sous-traitants ; direction DD et opinion } \\
\text { des répondants sur la RSE }\end{array}$ \\
\hline $\begin{array}{l}\text { Exploitation des don- } \\
\text { nées }\end{array}$ & $\begin{array}{l}\text { - Recours à l'analyse thématique } \\
\text { - Double analyse des données : verticale et horizontale. } \\
\text { - Analyse verticale : } \\
\text { - regroupement des informations collectées pour chaque enseigne } \\
\text { (entretiens + étude documentaire) } \\
\text { - synthèse par entretien et synthèse globale pour chaque enseigne } \\
\text { - informations classées par thèmes, sous-thèmes (définis a priori) } \\
\text { recherche des liens entre les thèmes } \\
\text { - Analyse horizontale : } \\
\text { - comparaison des enseignes sur la base des thèmes et sous-thèmes } \\
\text { (voir les similitudes/différences) } \\
\text { - synthèse des résultats } \\
\text { - Traitement manuel des données (nombre réduit d'entretiens) }\end{array}$ \\
\hline $\begin{array}{l}\text { Validité externe de la } \\
\text { recherche }\end{array}$ & $\begin{array}{l}\text { Mobilisation de plusieurs sources d'information : étude documentaire } \\
\text { et entretiens (principe de triangulation des données) ; pour chaque en- } \\
\text { seigne entretiens avec au moins deux dirigeants (obtenir des éclairages } \\
\text { différents) }\end{array}$ \\
\hline
\end{tabular}

2 Xerfi/France (septembre 2010). Distribution de meubles, Perspectives du marché à l'horizon 2011, Paysage concurrentiel et stratégies d'adaptation des acteurs : http://www.xerfi.com/etudes. 


\subsection{Présentation des entreprises étudiées}

La création de l'enseigne E1 remonte à une quarantaine d'années. Elle travaille avec un peu moins de 700 sous-traitants à travers le monde et appartient encore au début de notre étude à un groupe français coté en bourse. En 2010 l'enseigne a été vendue à un groupe international. E1 est présente en France ainsi qu'à l'étranger (avec plus de 230 magasins) et spécialisée dans «l'équipement du foyer ».

Depuis la mise en place d'une charte éthique vers la fin des années 90, la politique RSE du groupe n'a cessé de se structurer et de se développer : création d'un département Développement durable (DD) pour E1 en 2003 et d'une direction de la RSE pour le groupe en 2007 ; adhésion du groupe au Pacte mondial des Nations Unies en 2008.

Les sous-traitants proviennent majoritairement d'Europe de l'Ouest (58 \%) et d'Asie (38 \%). Ils doivent se conformer à la charte fournisseur qui s'appuie sur les grands principes éthiques énoncés dans le code de conduite des affaires du groupe (conçu en 2005). Il leur est également demandé de faire respecter ces principes auprès de leurs propres fournisseurs et soustraitants. Pour être retenu par l'enseigne un sous-traitant doit passer deux qualifications : fournisseur et produit. Trois conditions sont à remplir pour la qualification fournisseur : se plier à un audit technique en usine (réalisé par l'enseigne), à un audit social (sous-traité à un cabinet spécialisé et basé sur la grille ICS ${ }^{3}$ qui s’inspire de la norme sociale SA 8000) et signer la charte fournisseur.

La deuxième enseigne $\mathrm{E} 2$ a été créée il y a un peu plus de 30 ans et compte 147 points de vente. L'enseigne fait partie du canal de distribution « jeune habitat ». Elle est présente en France, Suisse et Espagne. Elle appartient au début de notre étude à un groupe familial français propriétaire de deux autres enseignes dans l'ameublement et d'une centrale d'achat. Pour cette étude nous nous sommes tournés vers un franchisé du groupe (groupe M) dirigeant 19 magasins de l'enseigne E2 dans un pays européen. Ce franchisé possède également toute une chaîne de grands magasins leader dans ce même pays. En 2014, le groupe familial français a été démantelé et les 19 magasins de l'enseigne E2 dans ce pays européen ont été rachetés par l'enseigne E1. Le groupe franchiseur français ne possède pas de direction du DD contrairement au groupe $\mathrm{M}$.

Le franchisé ne gère que $20 \%$ des sous-traitants (soit 15 sous-traitants), c'est-à-dire ceux qui l'approvisionnent en «spécificités » (produits adaptés au marché national). Nous n’avons pu interroger que ces sous-traitants qui sont tous européens. La partie code de conduite est gérée par le groupe franchiseur, mais celui-ci collabore avec le groupe $M$, qui possède une centrale d'achat en Asie, pour les approvisionnements et les audits. Afin d'améliorer l'application des normes fondamentales du travail, le groupe $\mathrm{M}$ collabore avec l'entreprise privée de

3 L'Initiative Clause Sociale (ICS) est un groupe issu du commerce et de la distribution réunissant des enseignes de la grande distribution. C'est un espace de concertation multiparties prenantes (enseignes, ONG, syndicats, salariés...). La grille ICS est le fruit de la réflexion de ce groupe et s'appuie sur les principales conventions et recommandations de l'OIT ainsi que sur les réglementations sociales de chaque pays. 
vérification STR Testing \& Inspection. Le franchisé s’appuie sur le fournisseur pour le respect des approvisionnements, des conditions de travail.

L'enseigne E3 est beaucoup plus récente que les précédentes. Elle a été créée à la fin des années 90 par son PDG actuel et a vu arriver deux nouveaux actionnaires importants en 2008.

C'est une enseigne d'ameublement-décoration. Elle compte au début de notre étude 198 magasins, est présente en France, mais aussi en Belgique, Espagne, Luxembourg et Italie.

Initiée en 2006 avec les fournisseurs de meubles indonésiens, la démarche RSE de E3 s'est ensuite étendue à l'Inde et à la Chine, ces trois pays représentant les principales sources d'approvisionnement pour l'enseigne. La direction du DD a été créée en 2010 et E3 est devenue signataire du Pacte mondial des Nations Unies en 2012.

E3 travaille de manière régulière avec 95 sous-traitants (95 \% sont asiatiques). En 2009, les fournisseurs ont dû se conformer à la charte envoyée par l'enseigne (remplacée en 2014 par un code de conduite). Pour contrôler sa mise en œuvre, E3 fait pratiquer des audits par The Forest Trust (TFT), expert dans les contrôles des chaînes d'approvisionnement, et par Business Trading Ethically (BTE) pour les audits sociaux. Dans son rapport RSE de 2014 l'enseigne dit s'appuyer sur le standard SA 8000 et sur la réalisation et le suivi des audits sociaux pour mieux accompagner ses fournisseurs.

\subsection{Les résultats synthétiques de l'étude qualitative}

Au regard des objectifs de cet article, les résultats de l'analyse thématique ont été regroupés en trois grands points abordés successivement.

\subsubsection{LES MOTIVATIONS, LES ATTENTES DES DONNEURS D'ORDRE FACE AUX DÉMARCHES RSE}

La recherche d'avantages fait partie des motivations qui prennent le plus d'importance (dans les discours des trois enseignes étudiées et dans les entretiens réalisés auprès des dirigeants) au côté de la réponse aux attentes/pressions des parties prenantes, de la recherche d'un plus grand engagement organisationnel (Tableau 3). Ces résultats contrastent avec d'autres études et notamment celle de Seuring et Müller (2008), la plus souvent reprise dans les articles traitant des politiques d'achats durables. Ils mettent en avant les facteurs déclencheurs suivants : la réglementation, les demandes des clients, la réponse aux parties prenantes. La recherche d'un avantage concurrentiel n'arrive qu'en quatrième position. Le rôle des valeurs de la culture organisationnelle n'est pas mentionné.

Les avantages mis en avant par les enseignes sont les suivants : éviter ou diminuer les risques d'image et de réputation, donner confiance au client - le fidéliser, améliorer la qualité des produits ainsi que les méthodes de fabrication et les matériaux, pérenniser les relations avec les fournisseurs. La démarche RSE permet d'apporter un avantage concurrentiel aux entreprises qui la mettent en place.

À travers leur démarche RSE les enseignes cherchent également à répondre aux attentes de leurs parties prenantes. Le tableau 3 montre qu'elles hiérarchisent leurs parties prenantes 
(Mitchell, Agle et Wood, 1997). Les clients constituent un groupe essentiel pour les trois enseignes françaises. Ces résultats sont à rapprocher de l'étude comparative réalisée par Maignan et Ralston (2002) : les firmes françaises insistent sur le rôle des clients dans les initiatives RSE. Les propos des responsables de E1 et E2 font ressortir une certaine pression de la part des consommateurs sur ces enseignes. Le fait que ces dernières soient des spécialistes de l'ameublement à prix bas, pour une clientèle à petit budget, les contraint dans leur démarche RSE, le client n'étant pas forcément prêt à payer plus cher un produit qui réponde à des critères RSE. L'enseigne E3, quant à elle, évoque une forte sollicitation de la part des ONG pouvant s'expliquer par sa communication, la publication de son engagement. Nos résultats toutefois divergent de ceux de Maignan et Ralston (2002) quant au rôle des parties prenantes réglementaires ${ }^{4}$ dans les initiatives RSE des entreprises françaises. Pour les trois enseignes, celles-ci n'arrivent qu'en dernière position dans le classement des parties prenantes les plus influentes. Cela peut s'expliquer, comme nous le verrons plus loin, par l'attitude proactive et volontariste de deux enseignes sur trois (E1 et E3). La réglementation ne décide pas de l'évolution de leurs politiques RSE ; elles ne sont pas dans une relation de dépendance (Gond et Igalens, 2008) et ne mènent pas des politiques réactives (Maignan, Hillebrand et McAlister, 2002).

Enfin, en ce qui concerne les attentes, la majorité des responsables interrogés ont spontanément évoqué les effets bénéfiques de la démarche RSE sur l'engagement des collaborateurs. Celle-ci peut permettre d'atteindre un plus grand engagement organisationnel; les membres de l'entreprise s'y sentant bien et étant fiers d'y travailler. Leurs discours vont dans le sens des apports de Maignan, Hillebrand et McAlister (2002) : la démarche RSE génère des liens entre l'entreprise et son personnel sur la base de valeurs et d'intérêts communs. Les enseignes attendent donc de la démarche qu'elle crée une forte appropriation de la culture d'entreprise, cette dernière influençant en retour l'engagement dans des activités socialement responsables.

Deux enseignes sur trois ont aussi mis l'accent sur le rôle majeur du leadership des dirigeants. Par leurs comportements, les actions menées, ils exercent une influence sur la culture organisationnelle de leur entreprise. Ce point est abordé par la directrice du DD de E1 lorsqu'elle évoque la première action forte en matière de RSE : l'acheminement par barge fluviale à la suite de la grève en 2001 de l'un des transporteurs routiers.

"À la base, la Direction transport a vu l'acheminement par barge fluviale comme un palliatif, mais ce sont des gens qui sont très convaincus en matière de développement durable. Très engagés en tant que citoyens ils y ont vu une opportunité professionnelle et en même temps l'intérêt de l'entreprise et en plus s'y sont retrouvés en termes de valeurs".

Ces dirigeants sont aussi à l'origine de la démarche de l'enseigne. La directrice du DD de l'enseigne E3 a également mis en avant l'engagement du PDG de l'enseigne dans les initiatives RSE : "La démarche est la volonté du PDG pour des raisons de convictions personnelles et un fort attachement aux artisanats traditionnels des pays en voie de développement. "

Le rôle des dirigeants apparaît donc comme central dans l'implantation des stratégies RSE et la mobilisation du personnel (Borchani Maktouf, 2010) pour les enseignes E1 et E3 contrairement à E2.

4 L'État et l'Union européenne par exemple font partie de ces parties prenantes réglementaires. 
Tableau 3. Synthèse des Facteurs explicatifs Des démarches RSE POUR LeS TROIS ENSEIGNES

\begin{tabular}{|c|c|c|c|}
\hline Facteurs & Enseigne E1 & Enseigne E2 & Enseigne E3 \\
\hline $\begin{array}{l}\text { Recherche } \\
\text { d'avantages }\end{array}$ & $\begin{array}{l}\text { - Aider à vendre l'entre- } \\
\text { prise aux clients } \\
\text { - Promesse aux clients : } \\
\text { produits fabriqués } \\
\text { dans de bonnes condi- } \\
\text { tions } \\
\text { - Réduction des risques } \\
\text { - Progression des pro- } \\
\text { ducteurs en termes de } \\
\text { compétences } \\
\text { - Effets bénéfiques sur } \\
\text { les méthodes de fabri- } \\
\text { cation, les matériaux, } \\
\text { la qualité des produits } \\
\text { - Diminution des prix } \\
\text { des produits achetés }\end{array}$ & $\begin{array}{l}\text { - Rester dans le jeu. } \\
\text { Choix stratégique à } \\
\text { moyen et long terme } \\
\text { - Répondre aux besoins } \\
\text { du consommateur } \\
\text { (société qui évolue) } \\
\text { - Entreprise mieux } \\
\text { appréciée, comprise, } \\
\text { notamment de ses } \\
\text { fournisseurs }\end{array}$ & $\begin{array}{l}\text { - Fidéliser une clientèle } \\
\text { avertie, citoyenne } \\
\text { - Donner confiance au } \\
\text { consommateur } \\
\text { - Pérenniser les relations } \\
\text { avec les fournisseurs } \\
\text { - Meilleure transpa- } \\
\text { rence sur la chaîne } \\
\text { d'approvisionnement } \\
\text { - Meilleure compré- } \\
\text { hension des enjeux } \\
\text { de fabrication d'un } \\
\text { produit } \\
\text { - Proposition de pro- } \\
\text { duits plus haut de } \\
\text { gamme }\end{array}$ \\
\hline $\begin{array}{l}\text { Réponse aux } \\
\text { attentes des } \\
\text { parties pre- } \\
\text { nantes (PP) }\end{array}$ & $\begin{array}{l}\text { Impossibilité de se sous- } \\
\text { traire à ses PP }\end{array}$ & $\begin{array}{l}\text { Très important de } \\
\text { répondre rapidement aux } \\
\text { attentes des clients }\end{array}$ & \\
\hline $\begin{array}{l}\text { (PP les + impor- } \\
\text { tantes) }\end{array}$ & $\begin{array}{l}\text { Clients, collaborateurs, } \\
\text { fournisseurs }\end{array}$ & Clients, collaborateurs & $\begin{array}{l}\text { Fournisseurs, clients, } \\
\text { ONG }\end{array}$ \\
\hline $\begin{array}{l}\text { Pressions des } \\
\text { PP }\end{array}$ & $\begin{array}{l}\text { Démarche RSE } \\
\text { contrainte par clientèle à } \\
\text { petit budget } \\
\text { Pour les autres PP : } \\
\text { faible }\end{array}$ & Très faible & Forte (ONG) \\
\hline $\begin{array}{l}\text { Valeurs } \\
\text { Leadership des } \\
\text { dirigeants }\end{array}$ & $\begin{array}{l}\text { Origine démarche : } \\
\text { direction transport } \\
\text { (convictions, valeurs) }\end{array}$ & Non évoqué & $\begin{array}{l}\text { Volonté du PDG (convic- } \\
\text { tions personnelles + fort } \\
\text { attachement à l'artisanat } \\
\text { traditionnel) }\end{array}$ \\
\hline Réglementation & $\begin{array}{l}\text { Non évoquée spontané- } \\
\text { ment }\end{array}$ & Idem & Idem \\
\hline $\begin{array}{l}\text { Plus grand en- } \\
\text { gagement orga- } \\
\text { nisationnel }\end{array}$ & $\begin{array}{l}\text { - Levier de la motiva- } \\
\text { tion des salariés } \\
\text { - Sens en interne et } \\
\text { fierté d'appartenance } \\
\text { - Fidéliser une partie } \\
\text { des collaborateurs } \\
\text { (partage de valeurs, } \\
\text { attire ceux qui ont } \\
\text { envie de se retrouver } \\
\text { dans ces valeurs) } \\
\end{array}$ & $\begin{array}{l}\text { - Être mieux appréciée } \\
\text { de ses collaborateurs } \\
\text { - Asseoir la compagnie } \\
\text { pour laquelle les sala- } \\
\text { riés travaillent }\end{array}$ & $\begin{array}{l}\text { - Fédérer } \\
\text { - Engouement et senti- } \\
\text { ment de fierté } \\
\text { - Forte appropriation de } \\
\text { la culture d'entreprise } \\
\text { - Bénéfices immédiats } \\
\text { en interne }\end{array}$ \\
\hline
\end{tabular}




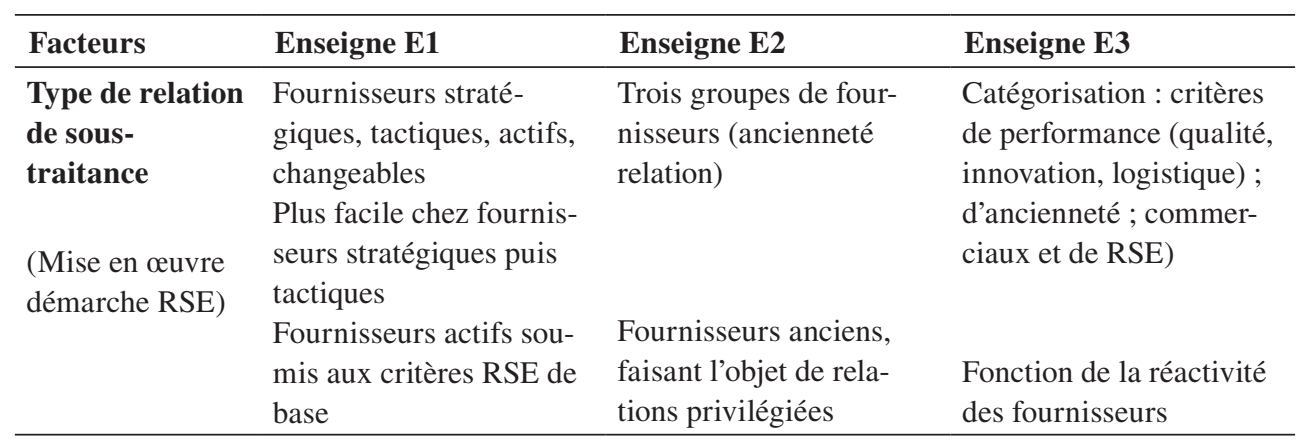

\subsubsection{RELATIONS DE SOUS-TRAITANCE ET DÉMARCHE RSE}

L'étude qualitative nous permet de constater que chaque DO effectue une classification de ses sous-traitants et la démarche RSE dépend du type de relation DO/sous-traitant (Tableau 3).

L'enseigne E1 réalise une typologie de ses sous-traitants, qualifiée de "star », en quatre catégories. Les « stratégiques » sont des sous-traitants de long terme, importants pour E1 du point de vue chiffre d'affaires et qui travaillent en partenariat avec l'enseigne. La mise en œuvre de la démarche RSE est plus aisée chez ces sous-traitants. Les " tactiques » ont un potentiel en termes de technologie, de capacité et peuvent devenir des sous-traitants «stratégiques ». Ces deux catégories sont considérées comme très performantes par le DO sur le plan de la qualité, de critères commerciaux ou logistiques. Les sous-traitants sont situés en Europe ou dans des pays en voie de développement. Les « actifs » n'ont pas d'avantages concurrentiels; ils sont substituables sur la base de critères prix ou commerciaux par exemple. Avec les « actifs ", l'enseigne s'implique au minimum : aucun programme, aucun plan de développement n'est effectué avec ces derniers. Ils sont soumis aux critères RSE de base. Les « changeables » sont des sous-traitants que l'enseigne ne gardera pas (critères RSE non atteints ou réclamations produits).

L'enseigne E2 effectue une classification de ses sous-traitants sur la base de l'ancienneté de la relation en trois groupes. Le premier groupe est composé de sous-traitants de long terme et avec lesquels les relations sont privilégiées (travail en commun sur le design, la qualité ou encore les ressources). Ils sont considérés comme stratégiques par l'enseigne. C'est en particulier avec l'un de ces sous-traitants que E2 développe des gammes de produits éco-conçus. Le deuxième groupe réalise pour le DO des produits nouveaux et ces sous-traitants sont moins anciens. Enfin, le troisième groupe est composé de PME qui démarrent leur relation avec le E2 et lui permettent de tester de nouveaux approvisionnements.

L'enseigne E3 catégorise ses sous-traitants sur la base de l'ancienneté, de critères de performance, de critères RSE (classification par pays), du chiffre d'affaires. Un sous-traitant est jugé stratégique pour l'enseigne lorsqu'il possède un savoir-faire et qu'il est performant en termes de rapport qualité-prix. La démarche RSE menée avec les sous-traitants dépend de leur réactivité face aux exigences RSE de l'enseigne. Celle-ci va alors développer avec eux d'autres critères RSE, éventuellement une double labellisation.

Les résultats de l'étude qualitative confirment donc l'importance d'inclure le type de relation de sous-traitance dans les facteurs explicatifs des politiques d'achats responsables et 
vont dans le sens des travaux déjà cités de Quairel (2006, 2007), Quairel et Auberger (2007), Leire et Mont (2010).

\subsubsection{LES CHANGEMENTS ORGANISATIONNELS ET DE STRUCTURE APPORTÉS PAR CES DÉMARCHES RSE CHEZ LES DONNEURS D'ORDRE}

Les entreprises étudiées se distinguent par leur niveau d'engagement plus ou moins élevé. Celui-ci se traduit par des pratiques, des dispositifs différents et s'accompagne de changements organisationnels et de structure plus ou moins conséquents. Or ceux-ci sont indispensables pour permettre l'intégration du développement durable (Lépineux, 2004). Ils s'observent à travers l'évolution de la structure dédiée à la RSE, la mise en place d'actions de formation et d'information en interne comme en externe, la prise en compte de critères RSE dans l'évaluation et la rémunération du personnel ou encore l'intégration dans la démarche RSE d'autres organisations.

E1 est l'enseigne la plus avancée en termes de démarche. Elle peut être qualifiée de proactive et sa stratégie RSE de "RSE intégrée » (Martinet et Payaud, 2008). Une entreprise proactive est une entreprise qui systématiquement anticipe et cherche à répondre aux demandes de ses parties prenantes (Maignan, Hillebrand et McAlister, 2002). Afin de mettre en œuvre sa politique RSE, l'enseigne s'est engagée dans des modifications structurelles et organisationnelles. Sa structure s'est étoffée à partir de 2003 de services dédiés à la responsabilité sociale, les préoccupations RSE sont intégrées dans toutes les fonctions de l'entreprise et la transversalité est recherchée afin de favoriser l'échange sur ces problématiques (comités RSE par branche ; réunions directions clés/direction RSE). La formation à la RSE concerne essentiellement les chefs de produits et les acheteurs, et l'évaluation ne vise que les dirigeants des branches (une part de leur rémunération variable dépend des critères RSE). L’enseigne informe et forme également ses sous-traitants à sa démarche RSE (site Internet, création d'une journée fournisseurs, intervention d'organismes extérieurs et déplacements dans les usines des sous-traitants). Elle les aide aussi à mettre en œuvre les objectifs RSE. E1 s'est d'autre part davantage ouverte sur l'extérieur (écoconception, adhésion de l'enseigne à l'ICS). Enfin, elle est l'enseigne la plus avancée en matière de diffusion d'informations sociales et environnementales avec la publication d'un rapport RSE au niveau du groupe. E1 est donc dans une démarche de responsabilisation de sa chaîne de valeur (Acquier, Daudigeos et Valiorgue, 2011) qui implique de coordonner un ensemble d'acteurs : sous-traitants, clients, autres distributeurs...

E2, quant à elle, adopte un comportement suiveur ; peu de changements sont constatés sur le plan organisationnel. On peut parler d'intégration faible des objectifs RSE dans la stratégie de l'entreprise (Capron et Quairel-Lanoizelée, 2007). On note tout d'abord l'absence de direction du DD au niveau du groupe franchiseur. La formation à la RSE, au sein de l'enseigne E2 à l'étranger, ne s'adresse qu'aux directeurs et seuls quelques objectifs sont pris en compte au niveau du groupe $\mathrm{M}$ dans la rémunération de certains dirigeants. Les fournisseurs sont informés principalement sur cette démarche grâce aux visites de l'enseigne.

Le degré d'ouverture sur l'extérieur pour la définition des objectifs RSE peut être qualifié de très limité (à l'exception du travail sur trois gammes de produits éco-conçus avec l'un de ses fournisseurs). Enfin, la diffusion d'informations sur la démarche RSE est faible (pas de 
rapport $\mathrm{DD}$, très peu d'informations à destination de la clientèle dans le catalogue ou sur le site Internet à propos de la démarche).

L'enseigne E3 n'en est qu'à ses débuts en termes de changements organisationnels, mais sa démarche peut être qualifiée de volontariste. La direction du DD date de 2010 et l'enseigne cherche à progresser rapidement. Elle souhaite placer les achats au cour de sa responsabilité. En interne, la formation sur la démarche RSE concerne principalement les acheteurs. La RSE n'est pas encore intégrée aux systèmes d'évaluation et de rémunération du personnel, la mise en place des indicateurs de progression ne datant que de 2010. Il est donc nécessaire d'avoir un certain recul en termes de réalisations. E3 s'appuie sur des ONG de terrain pour l'aider à former et accompagner ses fournisseurs dans sa démarche RSE, mais ces pratiques ne concernent encore que peu de fournisseurs. L'enseigne fait preuve d'une réelle volonté d'ouverture en recourant à ces ONG et en cherchant à entraîner d'autres acteurs de la distribution sur certains projets comme les projets de plantations communautaires (leur objectif est de protéger certaines essences de bois menacées de disparition et d'arriver à une certification FSC pour ces plantations). Avec l'aide d'ONG, E3 a également instauré des indicateurs de progression en matière sociale chez ses sous-traitants. La responsable DD souligne qu'en Indonésie par exemple seulement $4 \%$ de la population bénéficie d'une couverture sociale au lieu de $10 \%$ chez les salariés des sous-traitants travaillant pour E3. L'enseigne a pour objectif d'augmenter ce pourcentage. E3 s'est donc lancée dans un processus de responsabilisation de sa chaîne de valeur qui implique ses sous-traitants, les ONG avec lesquelles elle collabore, des acteurs de la distribution... L'enseigne E3 ne publie pas encore de rapport de DD (il faudra attendre 2014 pour la parution du premier rapport RSE), mais des informations sur son site et dans son catalogue sont validées par ses partenaires. Elle affiche par conséquent une volonté de diffusion d'informations sur sa démarche de transparence.

\section{PROPOSITION D'UN MODĖLE EXPLICATIF DES POLITIQUES D'ACHATS SOCIALEMENT RESPONSABLES}

Nous présenterons tout d'abord très brièvement les variables qui ont été ajoutées au modèle de Carter (2005), ensuite le modèle proposé, puis l'opérationnalisation des variables dans le questionnaire fournisseurs.

L'étude qualitative a confirmé l'importance de trois facteurs explicatifs de l'engagement des entreprises en matière de RSE dans les achats retenus par Carter et Jennings (2004), Carter (2005) : les pressions des clients, la culture organisationnelle et le leadership des dirigeants. Toutefois, l'impact de ce dernier facteur n'a pas été traité avec les sous-traitants et ne figure donc pas dans le modèle proposé. Cette première étude a également mis en avant deux autres variables non reprises dans le modèle théorique de départ (Carter, 2005) qui sont la recherche d'avantages et le type de relation donneur d'ordre/sous-traitant. 
Figure 1. Proposition D'Un MOdèLE EXPLiCATIF DES POLITIQUES D'ACHATS SOCIALEMENT RESPONSABLES

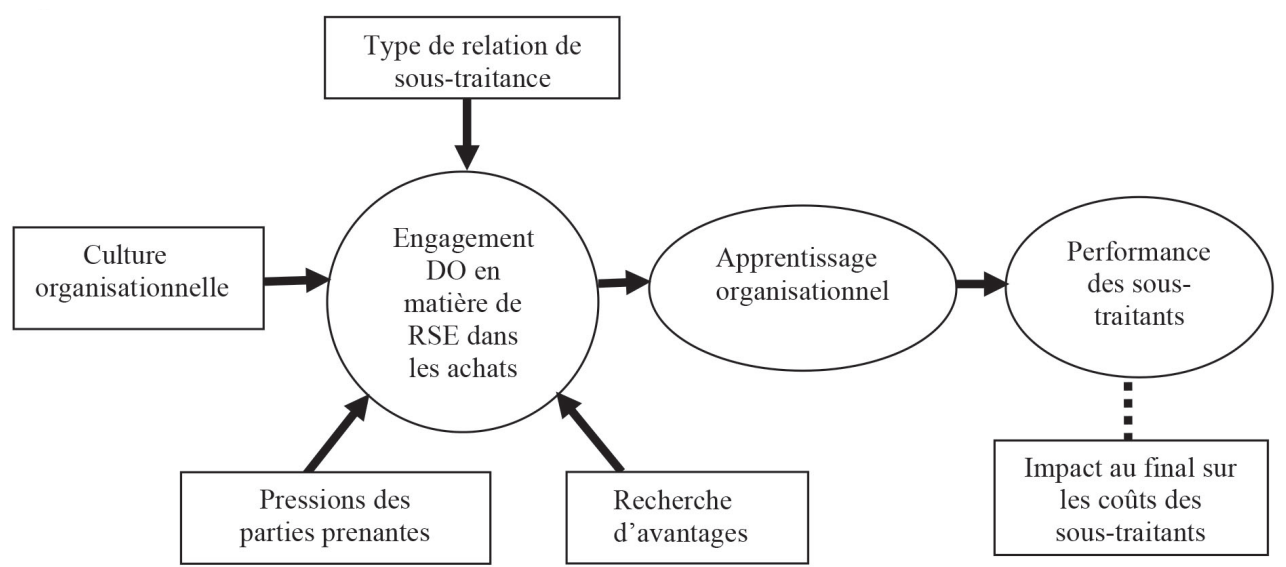

\subsection{L'opérationnalisation des variables dans le questionnaire fournisseurs}

Le mode opératoire mis en place par Carter et Jennings (2004), Carter (2005) n'a pu être repris dans le cadre du modèle proposé, la cible n'étant pas identique (sous-traitants dans notre étude, responsables achats pour ces auteurs). La performance des sous-traitants a été traitée ici comme un concept multidimensionnel. À côté des dimensions traditionnelles de la performance comme la qualité et les coûts, nous avons retenu d'autres critères comme l'innovation dans les produits, l'amélioration des méthodes de fabrication et des matériaux. La dimension sociale a également été prise en compte à travers l'amélioration des conditions de travail. Ces mesures de la performance se sont dégagées de nos entretiens menés avec les responsables des trois enseignes. Le tableau 4 présente la manière dont les différentes variables du modèle proposé ont été opérationnalisées. 


\begin{tabular}{|c|c|}
\hline Les variables du modèle & Opérationnalisation \\
\hline $\begin{array}{l}\text { Facteurs explicatifs de l'engagement des } \\
\text { entreprises en matière de RSE dans les } \\
\text { achats : } \\
\text { - les pressions des parties prenantes } \\
\text { - la recherche d'avantages } \\
\text { (amélioration performances écono- } \\
\text { miques ; réduction des risques) } \\
\text { - la culture organisationnelle } \\
\text { - le type de relation de sous-traitance }\end{array}$ & $\begin{array}{l}\text { (Question } 25 \text { avec échelle de Likert) } \\
\text { Questionnaire fournisseurs - Annexe } 1\end{array}$ \\
\hline $\begin{array}{l}\text { Engagement des entreprises en matière de } \\
\text { RSE dans les achats }\end{array}$ & $\begin{array}{l}\text { Évaluation du niveau d'engagement (question } 23 \\
\text { avec échelle d'attitude) }\end{array}$ \\
\hline Apprentissage organisationnel & $\begin{array}{l}\text { Hypothèse : une entreprise engagée en matière } \\
\text { de RSE dans les achats va former, auditer, aider } \\
\text { techniquement ses sous-traitants et permettre cet } \\
\text { apprentissage organisationnel (Carter, 2005). } \\
\text { La coopération avec les fournisseurs et les audits } \\
\text { font partie des critères retenus par Zhu, Sarkis et } \\
\text { Lai (2008) dans leur recherche portant sur le ma- } \\
\text { nagement vert de la chaîne d'approvisionnement. } \\
\text { Pour évaluer: } \\
\text { - la formation et l'aide technique reçues (questions } \\
\text { à échelle ordinale } 19 \text { et 20) } \\
\text { - les audits réalisés (question } 22 \text { à échelle) }\end{array}$ \\
\hline
\end{tabular}




\begin{tabular}{ll}
\hline Les variables du modèle & Opérationnalisation \\
\hline Performance des sous-traitants & Définition large de la performance (organisa- \\
& tionnelle, économique et sociale). Critères pris \\
& en compte pour son évaluation (question 26 avec \\
& échelle de Likert) : \\
& - l'amélioration de la qualité des produits \\
& - le développement de nouveaux produits \\
& - l'amélioration des méthodes de fabrication \\
& - l'amélioration des matériaux \\
& - l'amélioration des conditions de travail \\
\hline Coûts des sous-traitants & Impact de la RSE sur les coûts des sous-traitants \\
& (question 28, fermée unique) \\
\hline
\end{tabular}

Après avoir présenté le cadre conceptuel de la recherche, nous allons nous intéresser à présent à la réalisation de l'enquête par sondage. Avant d'analyser les résultats, la méthodologie sera brièvement exposée.

\section{LA PERCEPTION DES SOUS-TRAITANTS : L'ENQUÊTE PAR SONDAGE}

\subsection{La justification et la méthodologie de l'enquête par sondage}

Le but de cette enquête est de recueillir le point de vue des sous-traitants sur trois thématiques : la relation de sous-traitance, la démarche RSE du DO et les changements apportés par cette démarche. L'intégration du point de vue des sous-traitants - rarement pris en compte dans les études empiriques alors qu'ils sont aussi des protagonistes du phénomène étudié - permet d'analyser la dynamique de la relation $\mathrm{DO} /$ sous-traitants dans le cadre des politiques d'achats responsables. Il est intéressant de mettre en perspective ces résultats avec ceux de l'étude qualitative ; les deux phases qualitatives et quantitatives sont complémentaires.

L'enquête a été réalisée de janvier à juin 2010. Le questionnaire en anglais et en français a été envoyé par courriel à 160 sous-traitants (à tous les sous-traitants réguliers de l'enseigne E3, aux sous-traitants gérés spécifiquement par le franchisé de E2 à l'étranger et à un nombre limité de sous-traitants de $\mathrm{E} 1$ prélevés au hasard ${ }^{5}$ ). Au total, 55 entreprises ont répondu à l'enquête (22 sur 50 pour E1, 7 sur 15 pour E2 et 26 sur 95 pour E3), soit un taux de réponse de $34,4 \%$. Dans un premier temps, nous avons procédé à une analyse descriptive des résultats de l'enquête puis à une analyse bivariée ayant pour objectif de tester le modèle proposé.

$5 \quad$ Limitation du nombre de fournisseurs à contacter par le directeur de la centrale d'achats de E1 qui craignait de dévoiler la structure de ses fournisseurs. Choix d'une méthode d'échantillonnage probabiliste à partir du fichier fournisseurs. 
Nous avons fait appel au test du khi-deux pour étudier la distribution de variables prises deux à deux et leur lien éventuel (Tableaux 5 et 6).

Dans le cadre de cet article, les liens suivants sont étudiés : facteurs explicatifs des politiques d'achats responsables/engagement des entreprises en matière de RSE ; type de relation de sous-traitance/engagement des entreprises en matière de RSE (pratiques mises en œuvre) ; pratiques/performance des fournisseurs. Les principaux résultats de l'analyse descriptive et bivariée sont présentés dans le 4.2.

\subsection{L’analyse des résultats}

\subsubsection{L'ANALYSE DES RÉSULTATS DES FACTEURS EXPLICATIFS DES POLITIQUES D'ACHATS SOCIALEMENT RESPONSABLES}

L'analyse descriptive des résultats de l'enquête fournisseurs montre que les démarches RSE des DO sont guidées en premier par la recherche d'avantages $(68,3 \%$ des fournisseurs estiment que les démarches RSE sont guidées par un objectif de réduction des risques et $67,5 \%$ par un objectif d'amélioration des performances économiques), puis en deuxième par la culture organisationnelle des DO (pour 63,4 \% des fournisseurs). Un peu moins de $54 \%$ des fournisseurs estiment que les démarches RSE des DO s'expliquent par la pression des parties prenantes. Ces résultats vont dans le même sens que ceux de l'étude qualitative : la perception des sous-traitants sur les motivations des DO et les discours de ces derniers sont cohérents. L'intégration de la RSE dans les achats répond donc en premier, pour les soustraitants PME interrogés, à une logique économique. Cependant, les analyses bivariées ne permettent pas d'établir de liens entre les différents facteurs explicatifs énumérés ci-dessus et le niveau d'engagement des DO.

L'analyse des résultats d'autre part confirme le rôle du type de relation de sous-traitance pour l'intégration des PME dans la démarche RSE des DO. Une proposition de typologie est donnée en annexe 3. L'analyse bivariée a tout d'abord mis en évidence le lien entre l'aide technique apportée par les DO pour la mise en œuvre des objectifs RSE et le type de contrat Tableau 5, test du khi-deux). Dans leur grande majorité, les PME sous-traitantes qui travaillent à la commande, et donc qui peuvent être mises en concurrence à chaque fois, ne sont qu’occasionnellement, voire jamais aidées (18 fournisseurs sur 21). 
TAbleau 5. Test du Khi-deux. Lien Aide technique ApportéE/Type De CONTRAT

\begin{tabular}{llll}
\hline Type de contrat & $\begin{array}{l}\text { Aide technique } \\
\text { Jamais + occa- } \\
\text { sionnellement }\end{array}$ & $\begin{array}{l}\text { Aide technique } \\
\text { Fréquemment }\end{array}$ & Total \\
\hline & $\begin{array}{l}\text { Effectifs obser- } \\
\text { vés et entre } \\
\text { parenthèses } \\
\text { effectifs théo- } \\
\text { riques }\end{array}$ & & \\
\hline Contrat à la commande & $18(13,56)^{\text {a }}$ & $3(7,44)^{\text {b }}$ & $\mathbf{2 1}$ \\
\hline Contrat annuel & $4(6,46)$ & $6(3,54)^{\text {a }}$ & $\mathbf{1 0}$ \\
\hline Contrat de moyen terme + contrat de partenariat & $9(10,98)$ & $8(6,02)$ & $\mathbf{1 7}$ \\
\hline Total & $\mathbf{3 1}$ & $\mathbf{1 7}$ & $\mathbf{4 8}$ \\
\hline
\end{tabular}

khi-deux table (au seuil de 2,5\%) =7,38< khi-deux calculé (nombre de degrés de liberté $=2$ ) $=7,75$ $\rightarrow$ Le type de contrat et l'aide technique apportée par les DO pour la mise en œuvre des objectifs RSE sont liés avec un risque d'erreur de $2,5 \%$

Légende :

a. Cases pour lesquelles l'effectif réel est nettement supérieur à l'effectif théorique.

b. Case pour laquelle l'effectif réel est nettement inférieur à l'effectif théorique.

Les tests réalisés montrent également que la démarche RSE des DO dépend de la localisation géographique des sous-traitants. La formation à la démarche RSE (Tableau 6, test du khi-deux) ainsi que les audits réalisés sont liés à l'origine des sous-traitants, les DO concentrant leurs efforts sur les PME asiatiques par rapport aux PME européennes en raison tout d'abord du risque de réputation, mais aussi parce qu'il est nécessaire de les faire progresser en matière de respect des droits de l'homme, de conditions de travail, de qualité des produits... On retrouve donc à la fois le " côté défensif » et «messianique » dans les démarches RSE des DO (Quairel, 2006). Enfin, il ressort de l'analyse bivariée que la formation à la démarche RSE dépend de la coopération sous-traitants/DO, les enseignes privilégiant les soustraitants déterminants. 


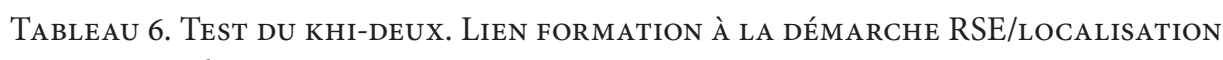
GÉOGRAPHIQUE DU SOUS-TRAITANT

\begin{tabular}{llll}
\hline Localisation géographique & $\begin{array}{l}\text { Formation } \\
\text { Jamais + occa- } \\
\text { sionnellement }\end{array}$ & $\begin{array}{l}\text { Formation } \\
\text { Fréquemment }\end{array}$ & Total \\
\hline & $\begin{array}{l}\text { Effectifs obser- } \\
\text { vés et entre } \\
\text { parenthèses } \\
\text { effectifs théo- } \\
\text { riques }\end{array}$ & & \\
& $21(24,29)$ & $14(10,71)$ & $\mathbf{3 5}$ \\
\hline Asie & $13(9,71)^{\mathrm{a}}$ & $1(4,29)^{\mathrm{b}}$ & $\mathbf{1 4}$ \\
\hline Europe & $\mathbf{3 4}$ & $\mathbf{1 5}$ & $\mathbf{4 9}$ \\
\hline Total & & & \\
\hline
\end{tabular}

khi-deux table (au seuil de $10 \%$ ) = 2,71 $<3,66$ (khi-deux calculé avec la correction de Yates) $<3,84$ (khi-deux table au seuil de $5 \%$ )

$\rightarrow$ La formation des fournisseurs à la démarche RSE dépend de leur localisation géographique, avec un risque d'erreur de $10 \%$

Légende :

a. Case pour laquelle l'effectif réel est nettement supérieur à l'effectif théorique.

b. Case pour laquelle l'effectif réel est nettement inférieur à l'effectif théorique.

\subsubsection{L'analyse des résultats liés aux changements apportés chez les sous-traitants}

L'analyse descriptive des résultats montre que pour l'ensemble des sous-traitants répondants, les démarches RSE des DO ont permis d'améliorer leur performance économique (la qualité des produits pour 75,6 \% des sous-traitants PME et le développement de nouveaux produits pour 57,5\%), leur performance organisationnelle (amélioration des méthodes de fabrication et des matériaux utilisés pour $56 \%$ ), mais aussi leur performance sociale (les conditions de travail pour $68,3 \%$ d'entre eux). Toutefois, des progrès restent à accomplir en matière de responsabilisation de la chaîne de valeur puisque $44 \%$ des sous-traitants seulement transmettent le code de conduite du DO à leurs propres fournisseurs.

Si l'on compare les résultats par enseigne, les avis sur les changements apportés par la démarche RSE sont mitigés chez les sous-traitants de E2 contrairement à ceux exprimés par les PME sous-traitantes des deux autres enseignes. Cela transparaît dans la perception qu'ils ont de l'engagement du DO (Tableau 7). Celle-ci est cohérente avec les pratiques RSE dans les achats pour les enseignes E1 et E2, mais semble en décalage pour E3. En effet, l'engagement de E1 se traduit bien par le déploiement de pratiques RSE et sa démarche est bien développée. De l'avis des sous-traitants, les valeurs et la culture jouent un rôle presque aussi important que la recherche d'avantages dans son engagement. Au contraire, pour E2, le degré de diffusion de la RSE est faible et l'engagement est jugé moyen. En ce qui concerne E3, la diffusion de la RSE est encore faible en raison d'une démarche récente, mais les sous-traitants évaluent favorablement l'engagement de l'enseigne E3. 
Tableau 7. Perception de l'engagement des DO et pratiques RSE dans les achats (\% DE SOUS-TRAITANTS)

\begin{tabular}{llll}
\cline { 2 - 4 } & E1 & E2 & E3 \\
\hline Perception engagement du DO & $\begin{array}{l}\text { Fort ou très fort } \\
70,6 \%\end{array}$ & $\begin{array}{l}\text { Moyen } \\
60 \%\end{array}$ & $\begin{array}{l}\text { Fort ou très fort } \\
61 \%\end{array}$ \\
\hline Information régulière $^{\text {a démarche RSE }}$ & $65 \%$ & $33 \%$ & $42 \%$ \\
\hline Audits (au moins 1 fois/an) & $69 \%$ & $20 \%$ & $53 \%$ \\
\hline Formation démarche RSE & Régulièrement & Jamais & Jamais \\
& $53 \%$ & $60 \%$ & $64 \%$ \\
\hline Aide technique & Aidés & Jamais aidés & Jamais aidés \\
& $58 \%$ & $67 \%$ & $66,5 \%$ \\
\hline
\end{tabular}

a. Regroupement des modalités «systématiquement », « très souvent » et « souvent ».

L'analyse descriptive fait aussi ressortir que pour 48,5\% des sous-traitants répondants, la démarche RSE a entraîné une augmentation de leurs coûts. Lors des questions ouvertes, certaines PME asiatiques ont mis en avant le dilemme entre la conformité à la RSE et leur compétitivité : «La mise en cuvre de la démarche est douloureuse et si nous n'avions pas été contraints par E3 nous ne l'aurions probablement jamais mise en cuvre. À côté de cela, en dehors de la destruction des marges, qui est malheureusement une tendance mondiale, nous percevons la politique de E3 comme une attitude positive dans la distribution européenne. Le point négatif est que la mise en cuvre de ces politiques a une influence sur les coûts, et rend difficile pour le fournisseur d'être à la fois compétitif et conforme à la RSE en même temps. »

Ils ont aussi évoqué la difficulté des pays en voie de développement, en raison d'un certain retard technique, à répondre aux exigences des $\mathrm{DO}$ sans que cela ne se répercute trop sur leurs coûts.

L'analyse bivariée quant à elle nous renseigne sur les pratiques permettant d'améliorer la performance économique, organisationnelle et sociale des sous-traitants PME ainsi que sur les entreprises les plus concernées par ces changements.

L'amélioration des conditions de travail et les progrès réalisés dans les matériaux utilisés sont liés à la formation et à l'aide technique apportées par les DO en matière de RSE. L'amélioration de la qualité des produits ainsi que celle des conditions de travail, mais aussi le développement de nouveaux produits sont liés aux audits réalisés. Ces pratiques, en particulier les audits, génèrent à l'intérieur de la relation de sous-traitance des apprentissages qui améliorent la performance économique, mais aussi sociale des sous-traitants. Les plans d'action mis en place à la suite de ces audits, en particulier au niveau des enseignes E1 et E3, permettent aux PME sous-traitantes de se corriger et de s'améliorer. Les audits, qui pourtant soumettent les sous-traitants au contrôle et à l'évaluation des DO, sont donc perçus par ces derniers comme des outils d'amélioration de leur performance.

Les tests réalisés nous ont également permis de constater que certains sous-traitants sont davantage concernés que d'autres par les changements apportés par la démarche RSE (Annexe 3) : les sous-traitants asiatiques, les plus récents, ceux qui coopèrent avec les DO 
et les PME réalisant plus de $10 \%$ de leur chiffre d'affaires avec les DO. Les démarches RSE bénéficient donc davantage aux sous-traitants à risque et déterminants (les plus concernés par les pratiques des DO). Lors des questions ouvertes, ce sont les sous-traitants récents et ceux d'origine asiatique qui ont émis les avis les plus positifs quant aux changements apportés par la démarche RSE des DO. Ils considèrent cette démarche comme un passage obligé pour pouvoir développer leur chiffre d'affaires et comme une opportunité de progrès. Les DO sont aussi beaucoup plus présents chez ces fournisseurs que ce soit par des audits techniques ou sociaux.

Enfin, l’analyse bivariée a confirmé le lien entre l'amélioration de la qualité des produits, des conditions de travail (pour la majorité des sous-traitants qui font état de ces améliorations) et l'augmentation de leurs coûts. Ces fournisseurs supportent donc une partie du coût de la démarche RSE.

\section{DISCUSSION}

L'étude de ces trois enseignes du secteur de l'ameublement montre que leurs démarches RSE dans les achats sont guidées en premier par la recherche d'avantages et par leur culture organisationnelle. Ces résultats diffèrent de ceux mis en avant par Seuring et Müller (2008), Maignan et Ralston (2002). Comme nous l'avons montré, les avantages économiques liés à une performance accrue des sous-traitants sous-tendent les initiatives de diffusion de la RSE sur la chaîne d'approvisionnement. Ces initiatives favorisent l'apprentissage organisationnel qui en retour améliore la performance de certains sous-traitants. Cette diffusion est largement favorisée par la culture organisationnelle du DO.

Notre recherche fait aussi ressortir le rôle clé joué par le type de relation de sous-traitance dans les stratégies RSE. Elle confirme, à partir de deux voies d'accès au terrain (une étude qualitative auprès des DO et une étude par sondage auprès de leurs sous-traitants) l'importance d'inclure cette variable comme facteur explicatif des politiques d'achats responsables et va dans le sens des rares travaux portant sur cette thématique (Quairel, 2006, 2007 ; Quairel et Auberger, 2007 ; Leire et Mont, 2010). Elle montre de plus concrètement quels sont les sous-traitants plus ou moins concernés par les pratiques RSE des DO (fournisseurs déterminants et à risque). Les DO s'adaptent à la complexité de leur réseau de sous-traitants et intègrent plus ou moins ces derniers dans leur démarche en fonction de l'importance qu'ils leur accordent. Toutefois l'étude qualitative a révélé, avec le cas de l'enseigne E3 (dont la démarche a été qualifiée de volontariste), que les enseignes pouvaient s'engager davantage avec des PME sous-traitantes qui ne sont pas forcément déterminantes, mais s'avèrent réactives aux initiatives RSE. Comme le soulignent Quairel et Auberger (2007), si le DO et le fournisseur sont tous deux proactifs en matière de RSE, un «partenariat constructif »va se mettre en place menant les deux entreprises à une démarche de progrès. L'engagement en matière de RSE est donc en mesure d'agir sur la relation DO/sous-traitant, même si ce dernier n'est pas a priori considéré comme important pour le DO.

Cet article met en avant les différences importantes existant entre les enseignes sur le plan des dispositifs mis en ouvre et des changements organisationnels. L'une d'elles ne semble pas avoir apporté de modifications conséquentes alors que les deux autres vont de l'avant en 
matière de RSE et adoptent une démarche proactive. En effet les enseignes E1 et E3 cherchent à coordonner un ensemble d'acteurs ${ }^{6}$ imbriqués dans une chaîne de valeur (Acquier, Daudigeos et Valiorgue, 2011 ; Vercher, 2010). L'exemple de E3 montre d'ailleurs, contrairement aux résultats de l'étude de Fel (2011), qu'une entreprise peut déployer une véritable stratégie RSE dans les achats sans que sa politique RSE ne soit mature. Cela transparaît au niveau des sous-traitants PME dans la perception, l'opinion qu'ils ont de l'engagement du DO. Cette étude confirme qu'une entreprise ne peut se contenter d'un simple affichage de sa démarche, mais doit engager de véritables changements organisationnels et de structure. Il en va de sa crédibilité auprès de ses parties prenantes externes comme ses fournisseurs, mais aussi internes comme ses collaborateurs.

Enfin, même si pour une majorité de PME les démarches RSE ont été source de progrès (principalement la qualité des produits et les conditions de travail), cette étude fait apparaitre que pour certaines d'entre elles il est très difficile de répondre à la fois aux exigences économiques et aux exigences RSE des DO. Pour 48,5 \%, la démarche RSE a entraîné une augmentation de leurs coûts. Si les DO exercent des pressions à la baisse sur les prix d'achat, la RSE est alors vécue comme une contrainte par les sous-traitants PME. Comme le soulignent Quairel et Auberger (2007), et nous rejoignons ici leur analyse, il est nécessaire que les DO révisent leur pression sur les prix afin de permettre à leurs fournisseurs de faire face à l'augmentation des coûts liée aux exigences RSE, mais aussi d'investir pour améliorer leurs performances économiques. Ce n'est qu'à cette condition que la démarche RSE pourra être un facteur de progrès.

\section{CONCLUSION}

Outre le fait que ce travail porte sur une thématique peu traitée dans la littérature, l'apport essentiel de notre recherche est d'avoir abordé la sous-traitance responsable à partir de deux voies d'accès au terrain (étude qualitative et étude quantitative) et intégré le point de vue des sous-traitants rarement pris en compte dans les études empiriques. La plupart des travaux souffrent d'un «tropisme donneur d'ordre " (Frigant, 2012). Seule la mise en perspective des résultats des deux études permet d'étudier la dynamique des relations $\mathrm{DO} /$ sous-traitants dans le cadre des politiques d'achats responsables.

Les apports de cet article seront présentés sous deux angles : théorique et managérial. Sur le plan théorique tout d'abord, le premier apport de cette recherche est d'avoir proposé un modèle explicatif des politiques d'achats responsables en privilégiant la dimension sociale. La recherche reste dominée par les préoccupations environnementales et les aspects sociaux ainsi que l'intégration des trois dimensions sont rares (Quairel et Auberger, 2007 ; Müller et Seuring, 2008). Ce modèle a été partiellement validé. Le deuxième apport théorique réside dans le fait que la performance a été traitée comme un concept multidimensionnel et étudiée du point de vue des sous-traitants. Cet aspect est fondamental lorsque l'on s'intéresse à la performance de la chaîne d'approvisionnement (Pagell, Krumwiede et Sheu, 2007). À côté des dimensions traditionnelles de la performance, d'autres critères ont été retenus comme

6 Sous-traitants, fournisseurs des sous-traitants, ONG, autres acteurs de la distribution, clients. 
l'innovation dans les produits, l'amélioration des méthodes de fabrication et des matériaux. Nous avons également interrogé les sous-traitants sur la transmission des exigences RSE du DO auprès de leurs propres fournisseurs (code de conduite) afin de juger de l'amélioration des conditions de travail dans la chaîne d'approvisionnement. Sur le plan managérial, cette recherche apporte tout d'abord un éclairage sur les pratiques qui permettent d'améliorer la performance des sous-traitants. Cette thématique est peu étudiée dans la littérature, les auteurs passant principalement en revue les pratiques qui révèlent une attitude proactive dans la gestion des relations avec les fournisseurs (Maignan, Hillebrand et McAlister, 2002) ou «les bonnes pratiques». Ainsi que nous l'avons mis en avant, les audits se révèlent être, du point de vue des sous-traitants PME, un outil performant d'augmentation de la performance (économique, mais aussi sociale) à condition qu'ils débouchent sur un suivi de ces derniers, sur des actions de formation, d'assistance et de conseil leur permettant de se corriger et de s'améliorer. L'analyse en profondeur de trois cas d'autre part nous a donné les moyens de comparer les pratiques, les dispositifs et les changements organisationnels d'enseignes situées à des stades différents de leur démarche RSE.

Le nombre réduit de questionnaires retournés par les sous-traitants ne nous a pas permis de généraliser les résultats ce qui représente la principale limite de notre étude. Une première perspective d'évolution de cette recherche serait donc d'étendre ce travail à un échantillon plus important d'entreprises de nationalités différentes et dans différents secteurs. Une autre piste de recherche consisterait à étudier plus en profondeur, en fonction des types de relations de sous-traitance, les stratégies RSE mises en œuvre par les DO, les réponses apportées par les sous-traitants aux exigences RSE, la prise en charge des coûts de la RSE et ceci de manière longitudinale. Ainsi, il serait possible de préciser quels sont les types de sous-traitants pour lesquels ces démarches RSE sont profitables. 
ANNEXE 1. QUESTIONNAIRE FOURNISSEURS EN FRANÇAis

\section{QUESTIONNAIRE FOURNISSEURS \\ VOTRE ENTREPRISE}

\section{Dans quelle zone géographique est située votre entreprise?}
Union européenne
$\bigcirc$ Amérique du Sud $\bigcirc$ Océanie
O Europe de l'Est, hors UE
○ Amérique du Nord
○ Autre (préciser)......
O Asie
$\bigcirc$ Afrique

2. Dans quel type de production êtes-vous spécialisé ?
Sièges
○ Petit mobilier
O Arts de la table
O Meubles de cuisine
L Literie
Textiles pour la maison
Meubles meublants
Luminaires
Si autres (préciser)

\section{VOTRE RELATION AVEC X}

3. Dans quel(s) type(s) de production êtes-vous fournisseur de $\mathbf{X}$ ? (Plusieurs réponses possibles)
Sièges
$\bigcirc$ Petit mobilier
O Arts de la table
Meubles de cuisine
Literie
$\bigcirc$ Textiles pour la maison
○ Meubles meublants
Électroménager
○ Si autres (préciser)...
○ Mobilier de jardin
$\bigcirc$ Luminaires

4. Depuis combien de temps travaillez-vous avec $X$ ?
Moins de 1 an
6-10 ans
○ $1-2$ ans
○ 11 ans et plus
O $3-5$ ans

5. Quelle a été la part de votre chiffre d'affaires réalisée avec X en 2008 ?
O Moins de $5 \%$
○ntre 10 et $20 \%$
O Non-réponse
Entre 5 et moins de $10 \%$
Plus de $20 \%$

6. Quel est le type de contrat qui vous lie à $\mathrm{X}$ ?
Contrat à la commande
Contrat de moyen terme
Contrat annuel
○ Contrat de partenariat (long terme)

7. Êtes-vous mis en concurrence par $\mathrm{X}$ avec d'autres fournisseurs?
○ Jamais
Occasionnellement
Souvent
0 Très souvent
○ Systématiquement 
8. Par rapport à $X$, vous vous qualifieriez de fournisseur ?
O Substituable
Stratégique
Important
Autre (préciser)

9. Sur quels critères pensez-vous avoir été sélectionné ?

\begin{tabular}{|c|c|c|c|c|c|}
\hline & $\begin{array}{l}\text { Pas du tout } \\
\text { important }\end{array}$ & $\begin{array}{l}\text { Peu } \\
\text { important }\end{array}$ & $\begin{array}{l}\text { Assez } \\
\text { important }\end{array}$ & Très important & $\begin{array}{l}\text { Extrêmem } \\
\text { important }\end{array}$ \\
\hline Prix & $\bigcirc$ & ○ & ○ & ○ & O \\
\hline Quantités & O & O & $\bigcirc$ & O & 0 \\
\hline Qualité & O & $\bigcirc$ & $\bigcirc$ & $\bigcirc$ & 0 \\
\hline Délais & O & O & $\bigcirc$ & O & 0 \\
\hline $\begin{array}{l}\text { Serviabilité, flexi- } \\
\text { bilité }\end{array}$ & O & O & ○ & O & 0 \\
\hline $\begin{array}{l}\text { Capacité d'inno- } \\
\text { vation }\end{array}$ & ○ & ○ & ○ & ○ & \\
\hline Critères RSE & O & ○ & 0 & 0 & \\
\hline
\end{tabular}

10. X pratique-t-il le système des enchères inversées sur Internet pour la sélection de ses fournisseurs?
O Oui
O Non

11. Si oui, avez-vous déjà été sélectionné de cette manière-là?
O Oui
Non

12. Pensez-vous que le degré de contrôle de $X$ sur ses fournisseurs est :
Très faible
○ Faible
$\bigcirc$ Moyen
Fort Très fort

13. Sur quels aspects portent les contrôles de $\mathrm{X}$ ?

Jamais Occasionnellement Souvent Très souvent Systématiquement

Respect des normes
de qualité produit
Respect des critères
de qualification
fournisseur
Critères RSE

$\begin{array}{ll}0 & 0 \\ 0 & 0 \\ 0 & 0\end{array}$


14. Participez-vous à la détermination des caractéristiques physiques et techniques des produits avec $\mathrm{X}$ ?
Jamais
Occasionnellement
$\bigcirc$ Souvent
Très souvent
Systématiquement

15. Comment qualifieriez-vous le degré de confiance dans vos relations avec $X$ ?
Très faible
Faible
Moyen
Fort
Très fort

16. Si le degré de confiance est " fort " ou " très fort ", quelles sont les variables qui l'expliquent?

Ordonnez ces variables en les numérotant de 1 (la plus importante) à 4 (la moins importante)

Les relations personnelles (avec les acheteurs, les responsables qualité de X...)

La communication

L'apprentissage en commun

Autre (préciser)

17. Si le degré de confiance est « très faible », « faible » ou « moyen » comment l'expliquezvous?

\section{LA DÉMARCHE DE RESPONSABILITÉ SOCIALE (RSE) DE X}

18. Êtes-vous informé de cette démarche RSE par X ?
$\bigcirc$ Jamais
O Occasionnellement
Souvent
Très souvent
Systématiquement

Si oui, de quelle(s) manière(s) ? (Plusieurs réponses possibles)
O Site Web
O Visites de X
○ournée fournisseur
$\bigcirc$ Autres (préciser)

19. Êtes-vous formé à cette démarche RSE par $\mathrm{X}$ ?
○ Jamais
Occasionnellement
○ Souvent
○ Très souvent
O Systématiquement

Si oui, de quelle(s) manière(s) ? (Plusieurs réponses possibles)
Organisme extérieur
V Visites de $\mathrm{X}$
Journée fournisseur
○ Autres (préciser) 
20. Recevez-vous une aide technique de la part de $\mathrm{X}$ pour la mise en œuvre des objectifs RSE ?
O Jamais
Occasionnellement
Souvent
$\bigcirc$ Très souvent
Systématiquement

21. Répercutez-vous les pratiques RSE $\left(^{*}\right)$ de X sur vos propres fournisseurs ?

${ }^{*}$ ) code de conduite, charte...
$\bigcirc$ Jamais
O Occasionnellement
$\bigcirc$ Souvent
$\bigcirc$ Très souvent
Systématiquement

22. Êtes-vous audité par, ou pour le compte de, $\mathrm{X}$ sur les critères RSE
Plusieurs fois par an
Une fois tous les 2 ans
$\bigcirc$ Une fois par an
O Moins d'une fois tous les 2 ans

23. Comment qualifieriez-vous l'engagement de X en matière de RSE ?
○ Très faible
○ Faible
Moyen
$\bigcirc$ Fort Très fort

24. L'engagement de X en matière de RSE par rapport à ses concurrents vous semble-t-il :
$\bigcirc$ Beaucoup moins important
Moins important
Aussi important
○ Plus important
$\bigcirc$ Beaucoup plus important

25. Pensez-vous que la démarche RSE de $\mathrm{X}$ auprès de ses fournisseurs répond :

$\begin{array}{lllll}\text { Pas du tout } & \begin{array}{l}\text { Plutôt pas } \\ \text { d'accord }\end{array} & \begin{array}{l}\text { Ni en accord, ni } \\ \text { en désaccord }\end{array} & \begin{array}{l}\text { Plutôt } \\ \text { d'accord }\end{array} & \begin{array}{l}\text { Tout à fait } \\ \text { d'accord }\end{array}\end{array}$

A un objectif de réduction des risques (risques de réputation, de perte de<smiles>O</smiles>
marché...)

A un objectif d'amélioration des performances économiques

Aux pressions de certaines parties prenantes (consommateurs, ONG...)

Aux valeurs, à la culture de X 


\section{CHANGEMENTS APPORTÉS PAR LA DÉMARCHE RSE DE X}

26. La démarche RSE vous a-t-elle permis?

$\begin{array}{lccccc} & \begin{array}{l}\text { Pas du tout } \\ \text { d'accord }\end{array} & \begin{array}{l}\text { Plutôt pas } \\ \text { d'accord }\end{array} & \begin{array}{c}\text { Ni en accord, ni } \\ \text { en désaccord }\end{array} & \begin{array}{l}\text { Plutôt } \\ \text { d'accord }\end{array} & \begin{array}{l}\text { Tout à fait } \\ \text { d'accord }\end{array} \\ \begin{array}{l}\text { D'améliorer la qualité de } \\ \text { vos produits }\end{array} & 0 & 0 & 0 & 0 & \bigcirc \\ \begin{array}{l}\text { De développer de nou- } \\ \text { veaux produits }\end{array} & 0 & 0 & 0 & 0 & 0 \\ \begin{array}{l}\text { D'améliorer vos mé- } \\ \text { thodes de fabrication }\end{array} & 0 & 0 & 0 & 0 & 0 \\ \begin{array}{l}\text { D'améliorer les matériaux } \\ \text { utilisés }\end{array} & 0 & 0 & 0 & 0 & 0 \\ \begin{array}{l}\text { D'améliorer les condi- } \\ \text { tions de travail dans votre } \\ \text { entreprise }\end{array} & 0 & 0 & 0 & 0 & 0\end{array}$

27. Par rapport à il y a 3 ans, vos marges bénéficiaires sont-elles aujourd'hui ?
$\bigcirc$ En augmentation
$\bigcirc$ En diminution
O Constantes
Non-réponse

28. La démarche RSE a-t-elle eu un impact sur vos coûts?
O Oui
O Non
Non-réponse

Si oui, vos coûts ont-ils :
○ugmenté
○ Diminué

29. Avez-vous noté une augmentation des contrôles de $X$ ?
O Oui
○ Non
Non-réponse

Si oui, cette augmentation est-elle liée à la démarche RSE ?
O Oui
○ Non

30. Pensez-vous que la démarche RSE de X est profitable ?
$\mathrm{O}$ A votre entreprise et à $\mathrm{X}$
A X seulement
$\bigcirc$ A votre entreprise seulement
$\bigcirc$ A aucun des deux

Pourquoi ? 


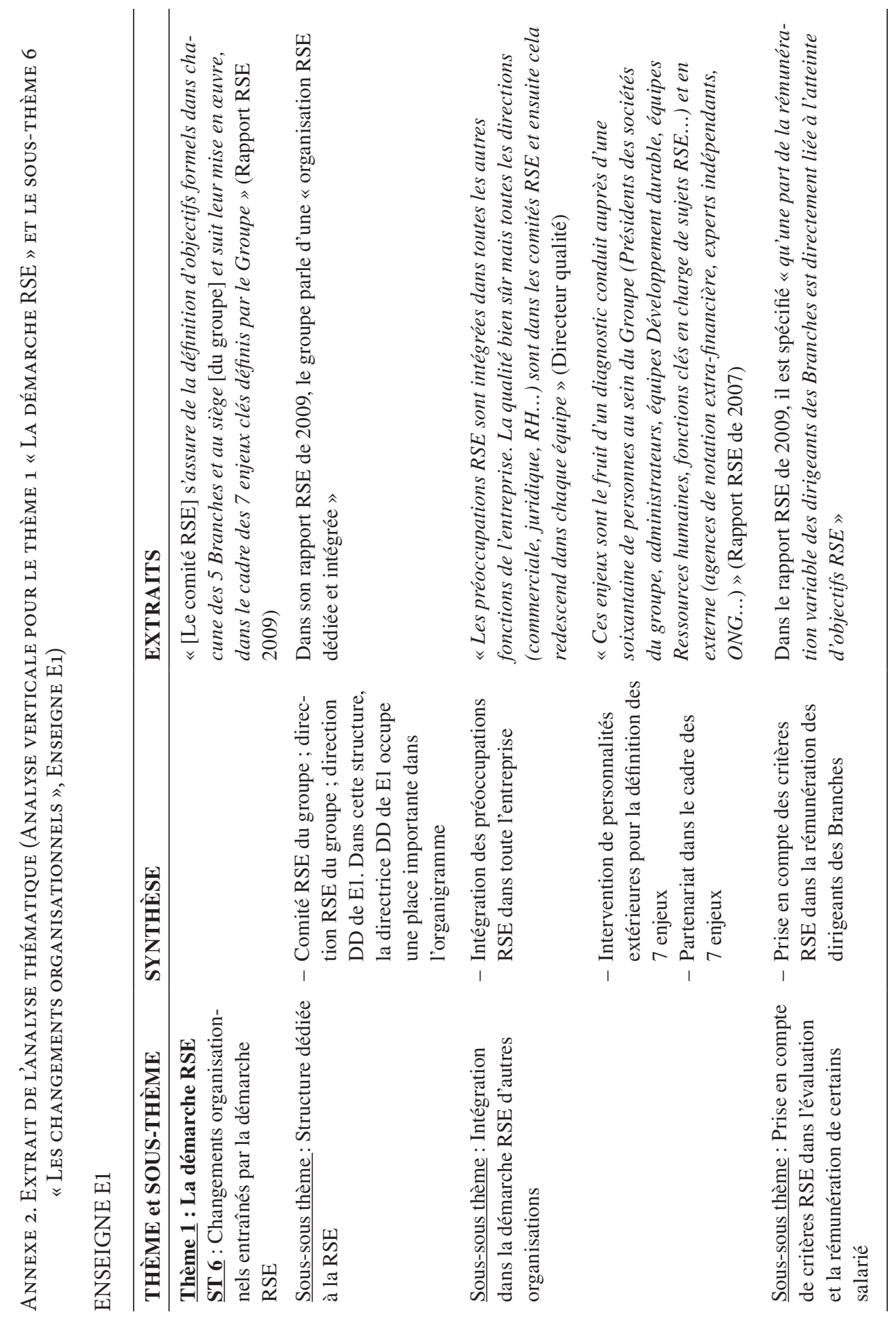




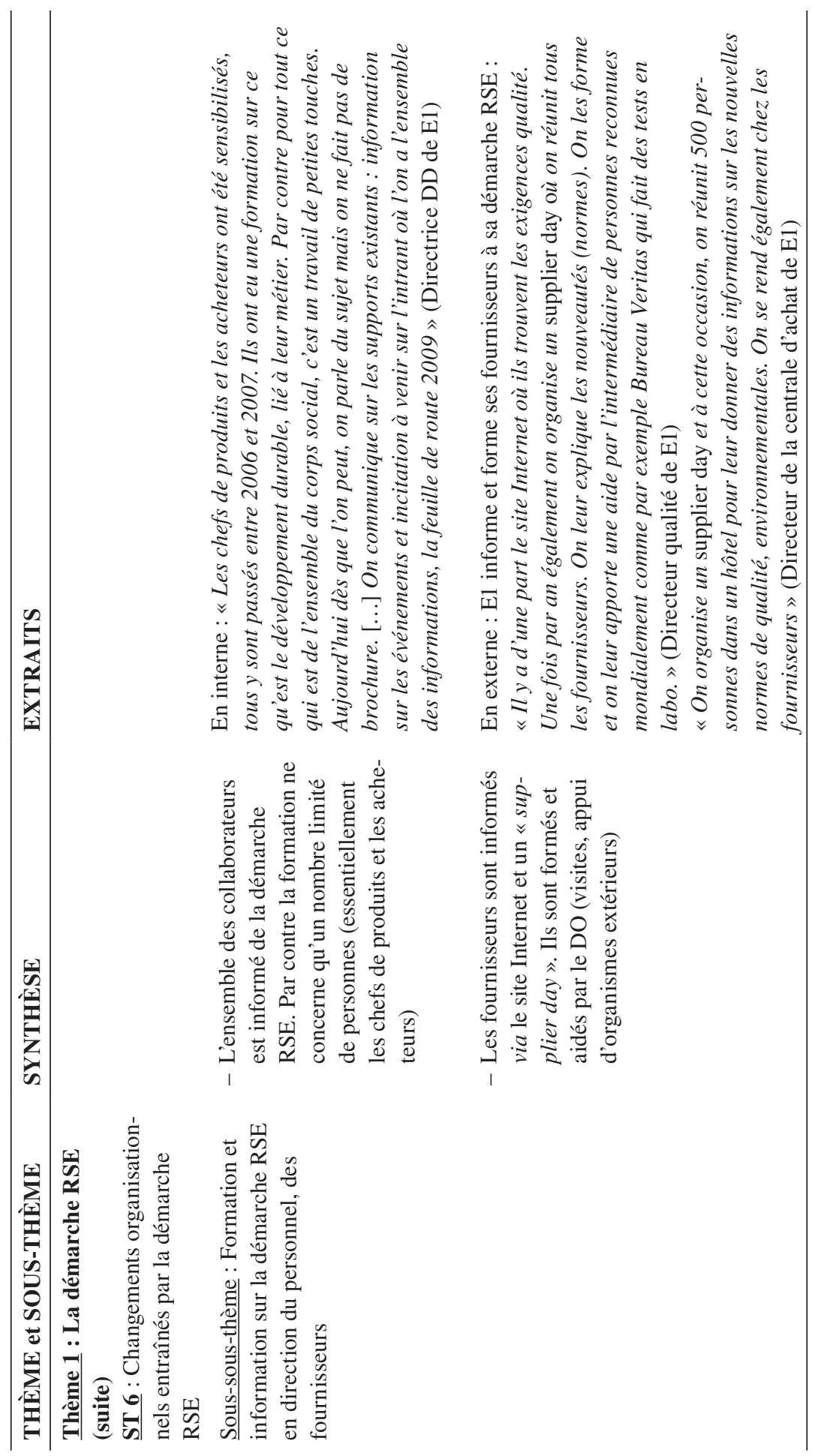




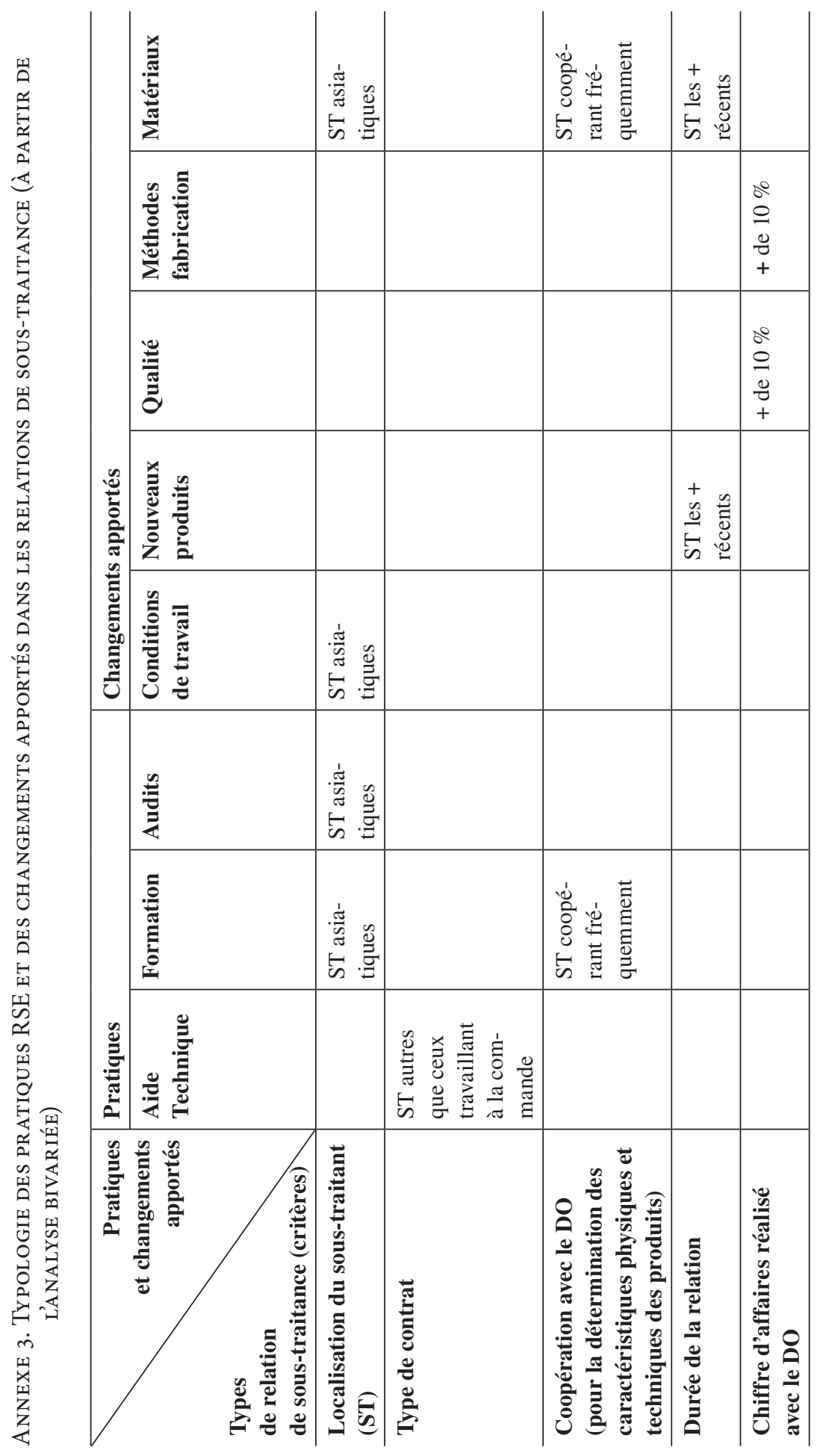




\section{RÉFÉRENCES}

Acquier, A. et Aggeri, F. (2008). Une généalogie de la pensée managériale sur la RSE. Revue française de gestion, 34(180), 131-157.

Acquier, A. Daudigeos, T. et Valiorgue, B. (2011). Responsabiliser les chaînes de valeur éclatées : enjeux et limites. Revue française de gestion, 37(215), 167-183.

ACKermann, R. et BAuer, R. (1976). Corporate social responsiveness : the modern dilemna. Virginie, Reston Publishing Company.

BARneY, J.B. (1991). Firm resources and sustained competitive advantage. Journal of Management, 17(1), 99-120.

BARney, J.B. (1999). How a firm's capabilities affect boundary decisions. Sloan Management Review, 40(3), 137-145.

BAUdry, B. (1993). Partenariat et sous-traitance : une approche par la théorie des incitations. Revue d'économie industrielle, 66, 51-68.

BAUdry, B. (1995). L'économie des relations interentreprises. Paris, La Découverte.

BAudry, B. et Chassagnon, V. (2012). Responsabilité sociale interfirmes, coordination et régulation de la firme-réseau multinationale : une analyse économique. Revue d'économie industrielle, 137, 43-64.

Belin-MUnier, C. (2009). Logistique, SCM et développement durable : une revue de la littérature. Atelier de recherche : transport et logistique : histoire(s) de durabilité. Récupéré le 17 septembre 2017 sur le site : http://www.let.fr/fr/atelier09/communications/belinmunier.pdf,2009.

Billaudot, B. et Julien, P.-A. (2003). Organisation industrielle et sous-traitance : du fordisme à l'entreprise-réseau. Dans P.A. Julien, L. Raymond, R. Jacob et G. Abdul-Nour (dir.), L'entreprise-réseau. Dix ans d'expérience de la chaire Bombardier Produits Récréatifs (p. 47-71). Sainte Foy, Presses de l’Université du Québec.

Borchani Maktouf, M. (2010). Déterminants de la stratégie sociétale des multinationales en France. Revue de l'organisation responsable, 5(2), 39-58.

Bowen, H.R. (1953). Social responsibilities of the businessman. New York, Harper \& Row.

Brandeburg, M., Govindan, K., Sarkis, J. et Seuring, S. (2014). Quantitative models for sustainable supply chain management : developments and directions. European Journal of Operational Resarch, 233(2), 299-312.

Brown, T.J. et DAcin, P.A. (1997), The company and the product : corporate associations and consumer product responses. Journal of Marketing, 61(1), 68-84.

Capron, M. et Quairel-Lanoizelée, F. (2007). La responsabilité sociale d'entreprise. Paris, La Découverte.

Capron, M. et Petit, P. (2011). Responsabilité sociale des entreprises et diversité des capitalismes. Revue de la régulation, (9). Récupéré le 23 mars 2016 sur le site : http://regulation.revues.org/9142.

Carter, C.R. (2004). Purchasing and social responsibility : a replication and extension. Journal of Supply Chain Management, 40(4), 4-16.

CARTer, C.R. (2005). Purchasing social responsibility and firm performance : the key mediating roles of organizational learning and supplier performance. International Journal of Physical Distribution \& Logistics Management, 35(3-4), 177-194. 
Carter, C.R. et Jennings, M. (2002). Social responsibility and supply chain relationships. Transportation Research Part E : Logistics and Transportation Review, 38(1), 37-52.

Carter, C.R. et Jennings, M. (2004). The role of purchasing in corporate social responsibility : a structural equation analysis. Journal of Business Logistics, 25(1), 145-186.

Commission européEnne (2001). Promouvoir un cadre européen pour la responsabilité sociale des entreprises. Livre vert.

De Woot, P. (2005). Responsabilité sociale de l'entreprise. Faut-il enchaîner Prométhée ? Paris, Economica.

Dupuis, J.-C. (2008). La responsabilité sociale de l'entreprise : gouvernance partenariale de la firme ou gouvernance de réseau ? Revue d'économie industrielle, 122, 67-86.

Evrard, Y., Pras, B. et Roux, E. (1998). Market. Études et recherches en marketing, connaître et pratiquer la gestion. Paris, Nathan.

FeL, F. (2011). Maturité des démarches RSE et achats durables. Revue Sciences de Gestion, 84, 83-100.

Freeman, R.E. (1984). Strategic management : a stakeholder approach. Boston, Marshall, M.A. Pitman.

Friedman, M. (1970). The social responsibility of business is to increase its profits. The New York Times Magazine, 13(32-33).

FRIGANT, V. (2012). Considérer les relations interfirmes pour comprendre l'adoption de pratiques socialement responsables : arguments à partir d'une critique du business case. Revue d'économie industrielle, 137, 65-84.

Gendron, C. (2000). Enjeux sociaux et représentations de l'entreprise. Revue du MAUSS, 15(1 $1^{\text {er }}$ trimestre), 320-325.

Gendron, C., Lapointe, A. et Turcotte, M.F. (2004). Responsabilité sociale et régulation de l'entreprise mondialisée. Relations industrielles, 59(1), 73-98.

Gond, J.P. et Igalens, J. (2008). La responsabilité sociale de l'entreprise. Paris, Presses universitaires de France.

Griffis, S.E., Autry, C.W., Thornton, L.M. et Brik, A.B. (2014). Assessing antecedents of socially responsible supplier selection in three global supply chain contexts. Decision Sciences, 45(6), 1187-1215.

Hajmohammad, S., Vachon, S., Klassen, R.D. et Gavronski, I. (2013). Reprint of lean management and supply management : their role in green practices and performance. Journal of Cleaner Production, 56, 86-93.

Leire, C. et Mont, O. (2010). The implementation of socially responsible purchasing. Corporate Social Responsibility and Environmental Management, 17(1), 27-39.

LÉPINeux, F. (2004). Dimension stratégique de la RSE et cohérence interfonctionnelle. Revue des Sciences de Gestion, 205, 75-92.

Levitt, T. (1958). The dangers of social responsibility. Harvard Business Review, 36(5), 41-50.

LoBEL, O. (2006). Sustainable capitalism or ethical transnationalism : offshore production and economic development. Journal of Asian Economics, 17(1), 56-62.

Maignan, I. et Ferrel, O.C. (2004). Corporate social responsibility and marketing : an integrative framework. Journal of the Academy of Marketing Science, 32(1), 3-19. 
Maignan, I., Hillebrand, B. et McAlister, D. (2002). Managing socially-responsible buying : how to integrate non-economic criteria into the purchasing process. European Management Journal, 20(6), 641-648.

Maignan, I. et Ralston, D. (2002). Corporate social responsibility in Europe and the US : insights from businesses' self-presentations. Journal of International Business Studies, 33(3), 497-514.

Marbach, C. (2007). "Le meuble en kit ». Service des études et des statistiques industrielles, Ministère de l'Économie, des Finances et de l'Industrie. Récupéré le 20 septembre 2017 sur le site : http://www. insee.fr/sessi/publications/etudes/mek/intro.pdf.

March, J.C. (1991). Exploration and exploitation in organizational learning. Organization Science, 2(1), 71-87.

Mariotti, F. (1999). L'apprentissage de la coopération dans l'entreprise-réseau (mémoire de DEA de génie industriel). Institut national polytechnique de Grenoble.

Mariotti, F., Reverdy, T. et Segrestin, D. (2001). Du gouvernement d'entreprise au gouvernement de réseau (rapport final). Commissariat général du Plan.

Martinet, A.C. et Payaud, M.A. (2008). Formes de RSE et entreprises sociales. Une hybridation des stratégies. Revue française de gestion, 34(180), 199-214.

Mitchell, R.K., Agle, B.R. et Wood, D.J. (1997). Toward a theory of stakeholder identification and salience : defining the principle of who and what really counts. Academy of Management Review, 22(4), 853-886.

Nogatchewsky, G. (2005). Seigneurie, vassalité, partenariat et marché dans les relations clientfournisseur. $14^{e}$ Conférence de l'Association internationale de management stratégique. Angers, juin. Récupéré le 20 septembre 2017 sur le site : http://www.strategie-aims.com/events/conferences/9-xiveme-conference-de-l-aims/communications/638-seigneurie-vassalite-partenariat-et-marche-dansles-relations-client-fournisseur.

Oliver, C. (1991). Strategic responses to institutional processes. Academy of Management Review, 16(1), 145-179.

ORSE/ECOVADIS (2010). Étude sur le reporting des grands groupes internationaux en matière d'achats responsables. Analyse de la communication externe de 125 entreprises asiatiques, américaines et européennes du Global 500. Paris.

Pagell, M., Krumwiede, D.W. et Sheu, C. (2007). Efficacy of environmental and supplier relationship investments. Moderating effects of external environment. International Journal of Production Economics, 45(9), 2005-2028.

Porter, M. et Kramer, M. (2006). Strategy and society : the link between competitive advantage and corporate social responsibility. Harvard Business Review, 84(12), 78-92.

Porter, M. et Kramer, M. (2011). The big idea : creating shared value. Harvard Business Review, $89(1-2), 62-77$

QuAIREL, F. (2006). La RSE aux frontières de l'organisation; entre ouverture et protection : exemple du management de la relation fournisseur dans les grands groupes industriels. Communication au

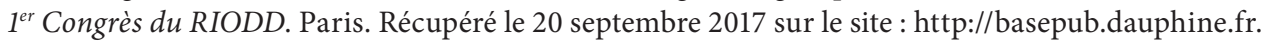

Quairel, F. (2007). Contrôle et RSE aux frontières de l'entreprise : la gestion responsable de la relation fournisseur dans les grands groupes industriels. $28^{e}$ Congrès de l'AFC, Comptabilité et environnement. Poitiers. Récupéré le 20 septembre 2017 sur le site : https://halshs.archives-ouvertes.fr/ halshs-00544956/document. 
Quairel, F. et Auberger, M.N. (2007). La diffusion de la RSE par la relation fournisseurs : injonctions paradoxales ou partenariat de progrès ? Revue internationale PME, 20(3-4), 69-94.

RoBERTS, S. (2003). Supply chain specific ? Understanding the patchy success of ethical sourcing initiatives. Journal of Business Ethics, 44(2-3), 159-170.

SALAm, A. (2009). Corporate social responsibility in purchasing and supply Chain. Journal of Business Ethics, 85(2), 355-370.

Segal, J.-P., Sobczak, A. et Triomphe, C-E. (2003). La responsabilité sociale des entreprises et les conditions de travail. Rapport pour la Fondation européenne pour l'amélioration des conditions de vie et de travail.

SEURING, S. (2013). A review of modeling approaches for sustainable supply chain management. Decision Support Systems, 54(4), 1513-1520.

Seuring, S. et Müller, M. (2008). From a litterature review to a conceptual framework for sustainable supply chain management. Journal of Cleaner Production, 16(15), 1699-1710.

Seuring, S., Sarkis, J., Müller, M. et RaO, P. (2008). Sustainability and supply chain management. An introduction to the special issue. Journal of Cleaner Production, 16(15), 1545-1551.

VACHON, S. et KLASSEN, R. (2008). Environmental management and manufacturing performance : the role of collaboration in the supply chain. International journal of production economics, 111(2), 299-315.

Vercher, C. (2010). Chaînes globales de valeur et responsabilité sociale des firmes de l'habillement. Le cas d'éthique sur l'étiquette. Revue française de gestion, 36(201), 177-193.

Worthington, I., Ram, M., Boyal, H. et ShaH, M. (2008). Researching the drivers of socially responsible purchasing : a cross-national study of supplier diversity initiatives. Journal of Business Ethics, 79(3), 319-331.

XeRFi/France (septembre 2010). Distribution de meubles, Perspectives du marché à l'horizon 2011, Paysage concurrentiel et stratégies d'adaptation des acteurs : http://www.xerfi.com/etudes.

YIN, R.K. (1984). Case study research : design and methods. Beverly Hills, Californie, Sage Publications.

Zhu, Q., SARKIS, J. et LAI, K. (2008). Confirmation of a measurement model for green supply chain management practices implementation. International journal of production economics, 111(2), 261-273. 